\section{Synthesis of 1,5-Anhydrohexitol Building Blocks for Oligonucleotide Synthesis}

Hexitol nucleic acids (HNA) represent a new oligomeric structure able to hybridize as well with DNA and RNA as with itself, and in a sequence-specific manner. In addition, HNA seems to be superior to its DNA and RNA analogs as an antisense construct. This is supported by a study demonstrating that HNA forms highly selective and exceptionally stable duplexes with RNA (Hendrix et al., 1997a) and that these HNA:RNA duplexes are stable towards nuclease degradation (Hendrix et al., 1997b). Furthermore, a high potential for antiviral activities has been reported (Verheggen et al., 1995).

Hexitol nucleic acids are made up of phosphorylated 1,5-anhydro-D-arabino-2,3-dideoxyhexitol building blocks with a base moiety positioned in the 2 position. According to the Westheimer model, the base moiety of the hexitol nucleosides is axially oriented, avoiding the sterically unfavorable 1,3-diaxial repulsions (De Winter et al., 1998).

This unit describes in detail the preparation of 1,5-anhydrohexitol (see Basic Protocol 1 and Fig. 1.9.1) and the 1,5-anhydrohexitol building blocks for oligonucleotide synthesis ( $h \mathrm{G}, h \mathrm{~A}, h \mathrm{C}, h \mathrm{~T}$; see Basic Protocols 2 to 5 and Figs. 1.9.2 to 1.9.5, respectively).

NOTE: Carry out reactions with anhydrous solvents and dried glassware (i.e., $2 \mathrm{hr}$ at $\left.70^{\circ} \mathrm{C}\right)$.

NOTE: All chemicals are commercially available (e.g., ACROS, Fluka, Aldrich). All starting materials can be synthesized but are also available commercially. The sugar intermediate $\mathbf{S . 5}$ is now also commercially available. All reactions can be carried out with standard laboratory equipment and glassware (e.g., round-bottom and Erlenmeyer flasks, consensers, dropping funnels, separatory funnels, desiccators, ultrasonic baths, oil baths, and magnetic stirrers).

\section{PREPARATION OF 1,5-ANHYDRO-4,6- $O$-BENZYLIDINE-3-DEOXY-D- GLUCITOL}

In this protocol, synthesis of the sugar building block starting from tetra- $O$-acetyl- $\alpha-D-$ bromoglucose is given, as outlined in Figure 1.9.1 (Verheggen et al., 1993). The starting sugar, tetra- $O$-acetyl- $\alpha$-D-bromoglucose, can be used to make both tolylsulfonyl and toluoyl intermediates (S.4a and $\mathbf{S . 4 b}$, respectively). The latter is used to make $\mathbf{S . 5}$ (described below), which is then used to make the $h \mathrm{G}, h \mathrm{~T}$, and $h \mathrm{C}$ nucleoside monomers (see Basic Protocols 2, 4, and 5, respectively). The former is used directly to make the $h$ A monomer (see Basic Protocol 3). The chemical reactions described give good yields, and purification of the intermediates is straightforward.

\section{Materials}

2,3,4,6-Tetra- $O$-acetyl- $\alpha$-D-bromoglucose

Diethyl ether: reflux overnight on sodium $\left(\mathrm{Na}, \mathrm{FeCl}_{2}, \mathrm{Et}_{2} \mathrm{O}\right)$ and distill

Azoisobutyronitrile [2,2'-azobis(2-methylpropionitrile); AIBN]

Tri- $n$-butyltin hydride

Precoated silica gel TLC plates (Alugram Sil G/UV254)

Dichloromethane

Anisaldehyde/sulfuric acid spray (UNIT 1.3)

Potassium fluoride dihydrate

Sodium sulfate

Silica gel $(0.060$ to $0.200 \mathrm{~nm})$

Methanol
BASIC

PROTOCOL 1

Synthesis of Modified

Nucleosides

\subsection{1}

Supplement 14 

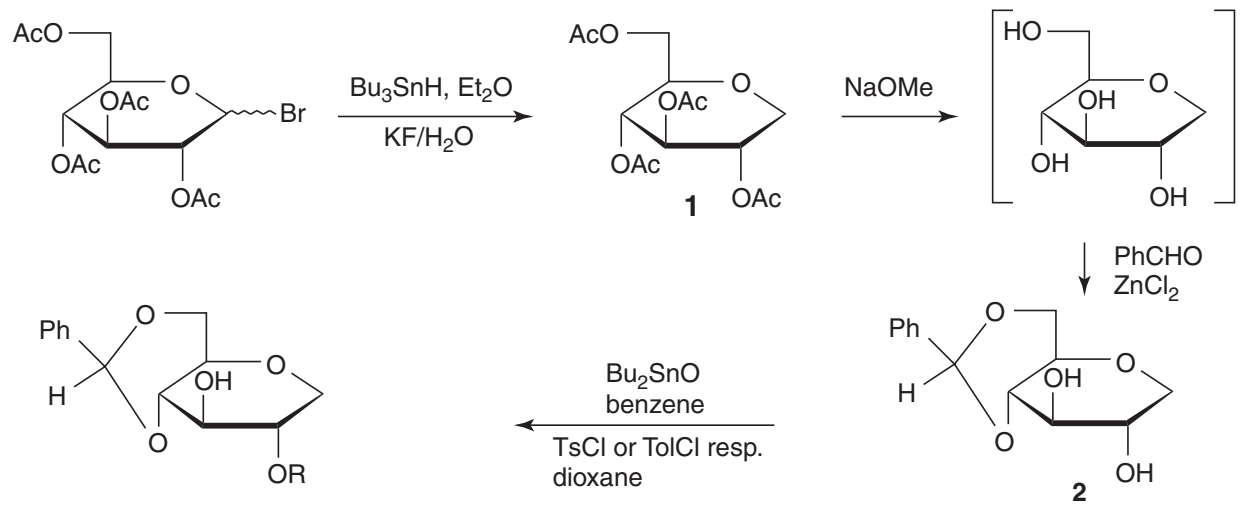

3a $\mathrm{R}=$ Tos

3b $\quad \mathrm{R}=\mathrm{Tol}$ $\mathrm{CSCl}_{2}$, DMAP, 2,4- $\mathrm{Cl}_{2} \mathrm{C}_{6} \mathrm{H}_{3} \mathrm{OH}, \mathrm{CH}_{2} \mathrm{Cl}_{2}$
$\mathrm{Bu}_{3} \mathrm{SnH}$, AlBN, toluene

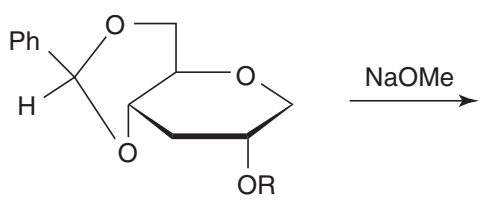

$$
\begin{array}{rl}
\mathbf{4 a} & \mathrm{R}=\mathrm{Tos} \\
\mathbf{4 b} & \mathrm{R}=\mathrm{Tol} \quad \square \mathrm{NaOMe} \\
\mathbf{5} & \mathrm{R}=\mathrm{H} \quad \longleftarrow \text { }
\end{array}
$$

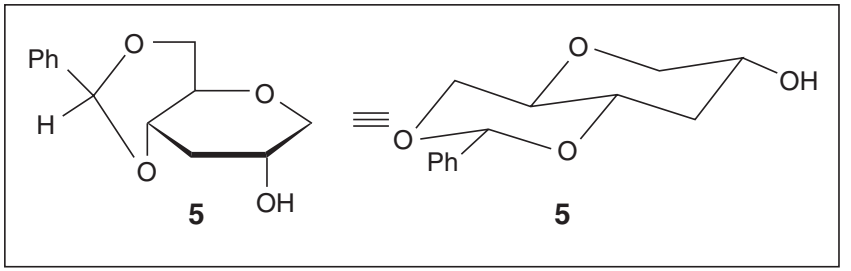

Figure 1.9.1 Preparation of 1,5-anhydro-4,6-O-benzylidine-3-deoxy-D-glucitol (S.5). Abbreviations: AIBN, azoisobutyronitrile; $\mathrm{Bu}_{2} \mathrm{SnO}$, dibutyltinoxide; $\mathrm{Bu}_{3} \mathrm{SnH}$, tributyltinhydride; DMAP, 4-(dimethylamino)pyridine; NaOMe, sodium methoxide; $\mathrm{PhCHO}$, benzaldehyde; $\mathrm{TolCl}$, toluoyl chloride; $\mathrm{TsCl}$, tosyl chloride.

Synthesis of 1,5-Anhydrohexitol Building Blocks
$0.1 \mathrm{~N}$ sodium methoxide, freshly prepared from sodium and dry methanol Acetic acid (glacial not necessary)

Toluene

Zinc chloride (dry fresh before use)

Benzaldehyde

Ethyl acetate

$n$-Hexane

Dibutyltinoxide

Benzene

Dioxane (reflux overnight on lithium aluminum hydride and distill)

$p$-Toluenesulfonyl chloride (for S.3a) or $p$-toluoyl chloride (for $\mathbf{S . 3 b}$ )

Delite Celite

4-(Dimethylamino)pyridine (DMAP)

Dry ice/isopropanol

Thiophosgene

2,4-Dichlorophenol

$1 \mathrm{M}$ potassium dihydrogenphosphate solution, $\mathrm{pH} 5$

Nitrogen gas

Oil bath and magnetic stirrer

Rotary evaporator equipped with a vacuum pump and cooling trap $5 \times 35-, 6 \times 50-$, and $5 \times 20-\mathrm{cm}$ chromatography columns 
$2 \times 18-\mathrm{cm}$ test tubes

1-L round-bottom flask with rubber stopper

Dropping funnel

Glass funnel

Dean-Stark condenser

UV lamp, $254 \mathrm{~nm}$

Additional reagents and equipment for thin-layer chromatography (TLC; APPENDIX

$3 D$ ) and column chromatography (APPENDIX 3E)

NOTE: The ${ }^{1} \mathrm{H}$ and ${ }^{13} \mathrm{C}$ NMR spectra given as examples were determined with a JEOL FX 90Q spectrometer or $400 \mathrm{MHz}$ Bruker AMX with tetramethylsilane as internal standard. Electron-impact mass spectra (EIMS) and chemical-ionization mass spectra (CIMS) were obtained using a KRATOS Concept ${ }^{1} \mathrm{H}$ mass spectrometer. Abbreviations: $\mathrm{s}$, singlet; d, doublet; dd, double doublet; t, triplet; br s, broad signal; m, multiplet; ddd, double doublet of doublet; and dm, double multiplet.

\section{Prepare S.1}

1. Dissolve $25.03 \mathrm{~g}(60.9 \mathrm{mmol})$ of 2,3,4,6-tetra- $O$-acetyl- $\alpha$-D-bromoglucose in $375 \mathrm{~mL}$ diethyl ether.

2. Add $0.99 \mathrm{~g}(6.1 \mathrm{mmol})$ of AIBN and $25 \mathrm{~mL}$ (93.1 mmol) of tri- $n$-butyltin hydride.

3. Stir the mixture $1 \mathrm{hr}$ at $35^{\circ} \mathrm{C}$ and then at room temperature until conversion is complete ( 2 to $12 \mathrm{hr}$ ). Monitor by TLC (APPENDIX $3 D$ ) by spotting the reaction mixture $(100 \mu \mathrm{L})$ between two spots of starting material $(1 \mathrm{mg}$ diluted to $200 \mu \mathrm{L})$ as a reference. Use precoated silica gel plates and develop with dichloromethane. Visualize under a UV lamp $(254 \mathrm{~nm})$ and then spray the plate with anisaldehyde/sulfuric acid spray and dry at $150^{\circ} \mathrm{C}$ (also see Critical Parameters).

4. Dissolve $8.21 \mathrm{~g}$ potassium fluoride dihydrate in $40 \mathrm{~mL}$ water, add to the suspension, and stir $15 \mathrm{~min}$.

5. Filter off the precipitated tri- $n$-butyltin hydride and wash the organic retentate (on top of the funnel) with $120 \mathrm{~mL}$ water. Set aside the aqueous layer and wash the organic layer two more times with $120 \mathrm{~mL}$ water. Combine all of the aqueous layers and wash three times with $60 \mathrm{~mL}$ diethyl ether.

6. Dry the combined organic layers from step 5 over sodium sulfate, filter, and evaporate to dryness using a rotary evaporator equipped with a vacuum pump and a cooling trap.

7. Divide the oily residue into two fractions and purify each by column chromatography (APPENDIX 3E) on $350 \mathrm{~g}$ of 0.060 - to $0.200-\mathrm{nm}$ silica gel in a $5 \times 35-\mathrm{cm}$ column. Use a step gradient from $1.5 \mathrm{~L}$ of $100 \%$ dichloromethane to $1.5 \mathrm{~L}$ of $99: 1(\mathrm{v} / \mathrm{v})$ dichloromethane/methanol to elute. Collect fractions in $2 \times 18-\mathrm{cm}$ test tubes.

See Critical Parameters for additional discussion of column chromatography.

8. Draw a roster of the fraction collector on a TLC plate and apply a drop of each fraction onto the corresponding field of the plate. Spray with anisaldehyde/sulfuric acid spray and dry at $150^{\circ} \mathrm{C}$. Combine the product-containing fractions (blue) and remove solvents in vacuo on a rotary evaporator.

The resulting product, 1,5-anhydro-2,3,4,6-tetra-O-acetyl-D-glucitol (S.1), should be obtained in a $93 \%$ yield $(18.82 \mathrm{~g}, 56.9 \mathrm{mmol})$. The spectroscopic properties are identical with those previously described (Kocienski and Pant, 1982).

Synthesis of Modified Nucleosides

\subsection{3}




\section{Prepare S.2}

9. Weigh $30.06 \mathrm{~g}$ (90.5 mmol) S.1 into a 1-liter round-bottom flask. Add $400 \mathrm{~mL}$ of $0.1 \mathrm{~N}$ sodium methoxide (freshly prepared from $0.92 \mathrm{~g}$ sodium and $400 \mathrm{~mL}$ dry methanol) and stir the reaction $2 \mathrm{hr}$ at room temperature.

10. Neutralize the reaction mixture with $\sim 2 \mathrm{~mL}$ acetic acid (confirm with $\mathrm{pH}$ paper) and evaporate the solvent.

11. Coevaporate the residue with $20 \mathrm{~mL}$ toluene four times. Add $12.40 \mathrm{~g}(91.0 \mathrm{mmol})$ zinc chloride and $46.50 \mathrm{~mL}$ (455.0 mmol) benzaldehyde. Close the reaction flask with a rubber stopper and vigorously stir the suspension for two days at room temperature.

12. Pour the reaction mixture into $250 \mathrm{~mL}$ ice-water and extract with $100 \mathrm{~mL}$ ethyl acetate four times. Dry the combined organic layers over sodium sulfate.

13. Remove excess benzaldehyde on a rotary evaporator in vacuo (bath temperature $70^{\circ} \mathrm{C}$ ) and wash the obtained solid residue with $100 \mathrm{~mL} n$-hexane on a glass funnel.

14. Purify by column chromatography using $700 \mathrm{~g}$ silica gel in a $6 \times 50-\mathrm{cm}$ column. Elute with a step gradient of $1.5 \mathrm{~L}$ each:

$1: 1(\mathrm{v} / \mathrm{v}) n$-hexane/dichloromethane

$100 \%$ dichloromethane

98:2 (v/v) dichloromethane/methanol.

Collect fractions in $2 \times 18-\mathrm{cm}$ test tubes and monitor by TLC as in step 8 . Combine product-containing fractions and remove solvent in vacuo on a rotary evaporator.

The resulting product, 1,5-anhydro-4,6-O-benzylidene-D-glucitol (S.2), is obtained in 75\% yield $(17.1 \mathrm{~g}, 68.0 \mathrm{mmol})$. CIMS $\left(\mathrm{iC}_{4} \mathrm{H}_{10}\right): \mathrm{m} / \mathrm{e} 253\left(\mathrm{MH}^{+}\right) ;{ }^{l} \mathrm{H}$ NMR $\left(\mathrm{DMSO}-\mathrm{d}_{6}\right): \delta=$ 3.00-3.90 ( $\mathrm{m}, 7 \mathrm{H})$ and 4.10-4.30 $(\mathrm{m}, 1 \mathrm{H})\left(H-\mathrm{l}^{\prime}, H-\mathrm{l}^{\prime \prime}, H-2^{\prime}, H-3^{\prime}, H-4^{\prime}, H-5^{\prime}, H-6^{\prime}, H-6^{\prime \prime}\right)$, 5.03-5.31 (dd, 2H, 2'-OH, 3'-OH), $5.55(\mathrm{~s}, \mathrm{lH}, \mathrm{PhCH})$, 7.20-7.57 (m, 5H, aromatic $\mathrm{H})$;

${ }^{13} \mathrm{C} \mathrm{NMR}\left(\mathrm{DMSO}-\mathrm{d}_{6}\right): \delta=68.0\left(C-6^{\prime}\right), 70.2,70.4\left(C-1^{\prime}, C-5^{\prime}\right), 71.0\left(C-2^{\prime}\right), 74.4\left(C-3^{\prime}\right)$, $81.1\left(\mathrm{C}-4^{\prime}\right)$, $100.7(\mathrm{PhCH}), 126.3,127.9,128.7,137.8$ (arom. C).

\section{Prepare S.3a or $S .3 b$}

15. Suspend $8.50 \mathrm{~g}(33.7 \mathrm{mmol}) \mathbf{S . 2}$ and $8.38 \mathrm{~g}(33.7 \mathrm{mmol})$ dibutyltinoxide in $250 \mathrm{~mL}$ benzene.

16. Reflux the mixture $16 \mathrm{hr}$ with azeotropic removal of water using a Dean-Stark condenser until the volume is reduced to $\sim 100 \mathrm{~mL}$. Add $150 \mathrm{~mL}$ dioxane.

17. For S.3a, add $7.06 \mathrm{~g}$ (37.0 mmol) $p$-toluenesulfonyl chloride and heat the mixture 6 $\mathrm{hr}$ at $50^{\circ} \mathrm{C}$, until a quantitative conversion to a less polar product (higher $R_{\mathrm{f}}$ value) is demonstrated by TLC. For $\mathbf{S . 3 b}$, add $4.44 \mathrm{~mL}(33.7 \mathrm{mmol}) p$-toluoyl chloride and stir the mixture $5 \mathrm{hr}$ at room temperature. For both, perform TLC using 1:2(v/v) $n$-hexane/dichloromethane and visualize the products under a UV lamp.

18. Concentrate the mixture in vacuo using a rotary evaporator, absorb on Delite Celite, and purify by column chromatography using a step gradient from $1: 1(\mathrm{v} / \mathrm{v}) n$-hexane/dichloromethane to $100 \%$ dichloromethane. Combine product-containing fractions and evaporate solvent on a rotary evaporator.

1,5-Anhydro-4,6-O-benzylidene-2-O-(p-tolylsulfonyl)-D-glucitol (S.3a) is obtained in 82\% yield $(11.22 \mathrm{~g}, 27.6 \mathrm{mmol})$. EIMS: $\mathrm{m} / \mathrm{e} 406\left(\mathrm{MH}^{+}\right) ;{ }^{1} \mathrm{H} N \mathrm{NMR}\left(400 \mathrm{MHz}, \mathrm{DMSO}-d_{6}\right): \delta=$ $2.42\left(\mathrm{~s}, 3 \mathrm{H}, \mathrm{CH}_{3}\right), 3.35-3.42\left(\mathrm{~m}, 2 \mathrm{H}, \mathrm{H}-4^{\prime}, \mathrm{H}-5^{\prime}\right), 3.49\left(\mathrm{t}, 1 \mathrm{H}, \mathrm{J}=11 \mathrm{~Hz}, \mathrm{H}-\mathrm{l}^{\prime} \mathrm{\alpha}\right), 3.61$ ( $\mathrm{m}$, $\left.1 \mathrm{H}, \mathrm{H}-\mathrm{\sigma}^{\prime} \alpha\right), 3.67\left(\mathrm{~m}, 1 \mathrm{H}, \mathrm{H}-3^{\prime}\right), 3.87\left(\mathrm{dd}, \mathrm{J}=5.5\right.$ and $\left.11 \mathrm{~Hz}, 1 \mathrm{H}, \mathrm{H}-\mathrm{l}^{\prime} \beta\right), 4.14-4.25(\mathrm{~m}, 2 \mathrm{H}$, $\left.H-2^{\prime}, H-6^{\prime} \beta\right), 5.05(\mathrm{~s}, 1 \mathrm{H}, \mathrm{PhCH}), 5.12(d, J=5.5 \mathrm{~Hz}, 1 \mathrm{H}, \mathrm{OH}), 7.35-7.50(\mathrm{~m}, 7 \mathrm{H}$, H-aromatic), 7.85 ( $\mathrm{m}, 2 \mathrm{H}$, H-aromatic); ${ }^{13} \mathrm{C} \mathrm{NMR}\left(90 \mathrm{MHz}, \mathrm{DMSO}-\mathrm{d}_{6}\right): \delta=21.0\left(\mathrm{CH}_{3}\right)$, 66.9, 67.6 (C-1, C-6), 70.7, 70.8 (C-3, C-5), 79.2, 80.4 (C-2, C-4), $100.7(\mathrm{PhCH}),+$ aromatic $C$. 
1,5-Anhydro-4,6-O-benzylidene-2-O-(p-toluoyl)-D-glucitol $(\mathbf{S . 3 b})$ is obtained in $78 \%$ yield $(9.73 \mathrm{~g}, 26.3 \mathrm{mmol})$. CIMS $\left(\mathrm{iC}_{4} \mathrm{H}_{10}\right): \mathrm{m} / \mathrm{e} 371\left(\mathrm{MH}^{+}\right) ;{ }^{1} \mathrm{H} N \mathrm{NMR}\left(\mathrm{DMSO}-\mathrm{d}_{6}\right): \delta=2.40,(\mathrm{~s}$, $3 \mathrm{H}, \mathrm{CH}_{3}$ ), 3.19-4.51 ( $\left.\mathrm{m}, 8 \mathrm{H}, \mathrm{H}-\mathrm{I}^{\prime}, \mathrm{H}-\mathrm{I}^{\prime \prime}, \mathrm{H}-2^{\prime}, \mathrm{H}-3^{\prime}, H-4^{\prime}, H-5^{\prime}, H-6^{\prime}, H-6^{\prime \prime}\right), 4.93-5.50(\mathrm{br}$, $\left.\mathrm{s}, 3^{\prime}-\mathrm{OH}\right), 5.55(\mathrm{~s}, 1 \mathrm{H}, \mathrm{PhCH}), 7.05-8.03(\mathrm{~m}, 9 \mathrm{H}, \mathrm{H}$-aromatic $) ;{ }^{13} \mathrm{C} \mathrm{NMR}\left(\mathrm{DMSO}-\mathrm{d}_{6}\right): \delta=$ $21.5\left(\mathrm{CH}_{3}\right), 67.2,68.4(\mathrm{C}-1, \mathrm{C}-6), 70.9,71.9,(\mathrm{C}-3, \mathrm{C}-5), 72.6(\mathrm{C}-2), 80.9(\mathrm{C}-4), 101.9$ $(\mathrm{PhCH}), 165.9(\mathrm{C}=\mathrm{O}),+$ aromatic $\mathrm{C}$.

\section{Prepare S.4a or $S .4 b$}

19. Dissolve $23.60 \mathrm{~g}$ (193.0 mmol) DMAP and either $11.22 \mathrm{~g}$ (27.6 mmol) S.3a or 10.21 $\mathrm{g}(27.6 \mathrm{mmol}) \mathbf{S . 3 b}$ in $400 \mathrm{~mL}$ dichloromethane.

20. Cool the reaction mixture to $-40^{\circ} \mathrm{C}$ with a dry ice/isopropanol mixture and add 2.53 $\mathrm{mL}(33.1 \mathrm{mmol})$ thiophosgene under vigorous stirring.

21. Allow the mixture to come slowly to room temperature and continue stirring $1 \mathrm{hr}$ at room temperature.

22. Add $6.30 \mathrm{~g}$ (38.6 mmol) 2,4-dichlorophenol and continue stirring another $2 \mathrm{hr}$. Monitor by TLC using 1:5 (v/v) $n$-hexane/dichloromethane and visualize with a UV lamp.

23. Pour the mixture into $300 \mathrm{~mL}$ of $1 \mathrm{M}$ potassium dihydrogenphosphate solution, $\mathrm{pH} 5$, and extract with $300 \mathrm{~mL}$ dichloromethane twice.

24. Dry the organic layers over sodium sulfate, filter, and evaporate the solvent.

25. Purify the residue using a $5 \times 35-\mathrm{cm}$ flash column and a step gradient from $1.5 \mathrm{~L}$ of $2: 8(\mathrm{v} / \mathrm{v}) n$-hexane/dichloromethane to $1.5 \mathrm{~L}$ of $100 \%$ dichloromethane. Combine product-containing fractions and evaporate solvent.

26. Dissolve the obtained thiocarbonyl compound in $300 \mathrm{~mL}$ toluene.

27. Bubble nitrogen gas through the solution for $10 \mathrm{~min}$ and then add $7.84 \mathrm{~mL}(29.1$ mmol) tri- $n$-butyltin hydride and $0.33 \mathrm{~g}(2.0 \mathrm{mmol})$ AIBN.

28. Heat the reaction mixture overnight at $80^{\circ} \mathrm{C}$.

29. Evaporate the mixture and purify by column chromatography as in step 25 .

1,5-Anhydro-4,6-O-benzylidene-3-deoxy-2-O-(p-tolylsulfonyl)-D-ribohexitol (S.4a) is obtained in 64\% yield $(6.90 \mathrm{~g}, 17.7 \mathrm{mmol})$. CIMS $\left.\left(\mathrm{NH}_{3}\right): \mathrm{m} / \mathrm{e} 391\left(\mathrm{MH}^{+}\right) ;{ }^{l} \mathrm{H} \mathrm{NMR}_{(\mathrm{CDCl}}\right)$ : $\delta=1.50-2.10\left(\mathrm{~m}, 2 \mathrm{H}, \mathrm{H}-3^{\prime}, \mathrm{H}-3^{\prime \prime}\right), 2.48\left(\mathrm{~s}, 3 \mathrm{H}, \mathrm{CH}_{3}\right), 3.06-4.84\left(\mathrm{~m}, 7 \mathrm{H}, \mathrm{H}-\mathrm{I}^{\prime}, \mathrm{H}-\mathrm{I}^{\prime \prime}, \mathrm{H}-2^{\prime}\right.$, $\left.H-4^{\prime}, H-5^{\prime}, H-6^{\prime}, H-6^{\prime \prime}\right), 5.50(s, 1 H, P h C H), 7.04-7.98$ ( $m, 9 H, H$ aromat.); ${ }^{13} \mathrm{C} \mathrm{NMR}$ $\left(\mathrm{CDCl}_{3}\right): \delta=21.4\left(\mathrm{CH}_{3}\right), 35.3\left(\mathrm{C}-3^{\prime}\right), 68.7,69.0\left(\mathrm{C}-\mathrm{I}^{\prime}, \mathrm{C}-6^{\prime}\right), 72.9,73.1\left(\mathrm{C}-4^{\prime}, \mathrm{C}-5^{\prime}\right), 75.7$ $\left(\mathrm{C}-2^{\prime}\right), 101.5(\mathrm{PhCH})+$ aromatic $C$.

1,5-Anhydro-4,6-O-benzylidene-3-deoxy-2-O-(p-toluoyl)-D-ribohexitol $(\boldsymbol{S . 4 b})$ is obtained in $75 \%$ yield $(7.12 \mathrm{~g}, 20.7 \mathrm{mmol})$. CIMS $\left(\mathrm{iC}_{4} \mathrm{H}_{10}\right): \mathrm{m} / \mathrm{e} 355\left(\mathrm{MH}^{+}\right) ;{ }^{1} \mathrm{H} \mathrm{NMR}\left(\mathrm{CDCl}_{3}\right): \delta=$ 1.42-2.12 ( $\left.\mathrm{m}, 2 \mathrm{H}, \mathrm{H}-3^{\prime}, \mathrm{H}-3^{\prime \prime}\right), 2.39\left(\mathrm{~s}, 3 \mathrm{H}, \mathrm{CH}_{3}\right), 3.12-3.92(\mathrm{~m}, 4 \mathrm{H})$ and $4.02-4.49(\mathrm{~m}, 2 \mathrm{H})$ $\left(H-l^{\prime}, H-1^{\prime \prime}, H-4^{\prime}, H-5^{\prime}, H-6^{\prime}, H-6^{\prime \prime}\right), 4.95-5.43\left(m, 1 H, H-2^{\prime}\right), 5.54(s, 1 H, P h C H)$, 7.10-8.08 (m, 9H, H aromat. $) ;{ }^{13} \mathrm{CNMR}\left(\mathrm{CDCl}_{3}\right): \delta=21.5\left(\mathrm{CH}_{3}\right), 34.8\left(\mathrm{C}-3^{\prime}\right), 66.9\left(\mathrm{C}-5^{\prime}\right)$, 69.0, 69.1 (C-1', C-6 $\left.6^{\prime}\right), 73.4\left(C-2^{\prime}\right), 76.0\left(C-4^{\prime}\right), 101.5(P h C H), 165.3(C=O)$, + aromatic C. 
Prepare S.5

30. Weigh $6.79 \mathrm{~g}$ (19.7 $\mathrm{mmol}) \mathbf{S . 4 b}$ into a 1-liter round-bottom flask.

31. Add $300 \mathrm{~mL}$ of $0.1 \mathrm{~N}$ sodium methoxide (freshly prepared from $0.70 \mathrm{~g}$ sodium and $300 \mathrm{~mL}$ dry methanol) and stir the reaction $4 \mathrm{hr}$ at room temperature.

32. Neutralize the reaction mixture with acetic acid $(\sim 6 \mathrm{~mL})$, confirming with $\mathrm{pH}$ paper, and evaporate the solvent.

33. Purify by flash chromatography using a $5 \times 20-\mathrm{cm}$ column and eluting with $99: 1$ $(\mathrm{v} / \mathrm{v})$ dichloromethane/methanol. Combine product-containing fractions and remove solvent in vacuo on a rotary evaporator.

The resulting product of 1,5-anhydro-4,6-O-benzylidene-3-deoxy-D-glucitol (S.5) is obtained in $80 \%$ yield $(3.72 \mathrm{~g}, 15.8 \mathrm{mmol})$. CIMS $\left(\mathrm{iC}_{4} \mathrm{H}_{10}\right): \mathrm{m} / \mathrm{e} 237\left(\mathrm{MH}^{+}\right) ;{ }^{1} \mathrm{H} \mathrm{NMR}$ $\left(\mathrm{DMSO}-\mathrm{d}_{6}\right): \delta=1.20-1.66\left(\mathrm{~m}, 1 \mathrm{H}, \mathrm{H}-3^{\prime}\right), 2.06-2.42\left(\mathrm{~m}, 1 \mathrm{H}, \mathrm{H}-3^{\prime \prime}\right), 2.99-3.98(\mathrm{~m}, 6 \mathrm{H})$ and 4.05-4.30 (m, $1 H)\left(H-1^{\prime}, H-l^{\prime \prime}, H-2^{\prime}, H-4^{\prime}, H-5^{\prime}, H-6^{\prime}, H-6^{\prime \prime}\right), 5.08$ (d, 1H, 2'-OH), $5.57(s$, $1 \mathrm{H}, \mathrm{PhCH}), 7.17-7.67$ ( $\mathrm{m}, 5 \mathrm{H}, \mathrm{H}$ aromatic $) ;{ }^{13} \mathrm{C} \mathrm{NMR}\left(\mathrm{DMSO}-\mathrm{d}_{6}\right): \delta=38.3\left(\mathrm{C}-3^{\prime}\right), 65.4$ $\left(C-5^{\prime}\right), 69.1\left(C-6^{\prime}\right), 72.3,73.0\left(C-1^{\prime}, C-2^{\prime}\right), 76.3\left(C-4^{\prime}\right), 101.6(P h C H), 126.1,128.2,129.0$, 137.2 (arom. $C$ ).

BASIC PROTOCOL 2

Synthesis of 1,5-Anhydrohexitol

Building Blocks

1.9.6

\section{SYNTHESIS OF $1^{\prime}, 5^{\prime}$-ANHYDRO-2', $3^{\prime}$-DIDEOXY-2' $-\left(N^{2}\right.$-ISOBUTYRYL-} GUANIN-9-YL)-6'- $O$-MONOMETHOXYTRITYL-D-ARABINOHEXITOL

This protocol details the synthesis of the $h \mathrm{G}$ 1,5-anhydrohexitol building block S.9 from S.5 (see Fig. 1.9.2 and DeBouvere et al., 1997).

\section{Materials}

6-Chloro-9H-purine-2-amine

1,5-Anhydro-4,6-O-benzylidene-3-deoxy-D-glucitol (S.5; see Basic Protocol 1)

Triphenylphosphine

Dioxane (reflux overnight on lithium aluminum hydride and distill)

Nitrogen gas

Diisopropyl azodicarboxylate (DIAD)

n-Hexane

Ethyl acetate

$10 \%(\mathrm{v} / \mathrm{v}) \mathrm{HCl}$

Dichloromethane (store over phosphorous pentoxide and distill before use)

Phenolpthalein solution

$4 \mathrm{~N}$ sodium hydroxide

Phosphorous pentoxide

Pyridine (reflux overnight over potassium hydroxide distill before use)

Bis(trimethylsilyl)acetamide (BSA)

Isobutyric anhydride

$25 \%$ (v/v) ammonia

Diethyl ether

Dimethylformamide (DMF; remove water by distillation with benzene followed by distillation under vacuum)

4-Monomethoxytrityl chloride (MMTr·Cl)

Methanol

Saturated sodium bicarbonate solution

Sodium sulfate

Toluene

Dropping funnel

Rotary evaporator equipped with a vacuum pump and cooling trap 


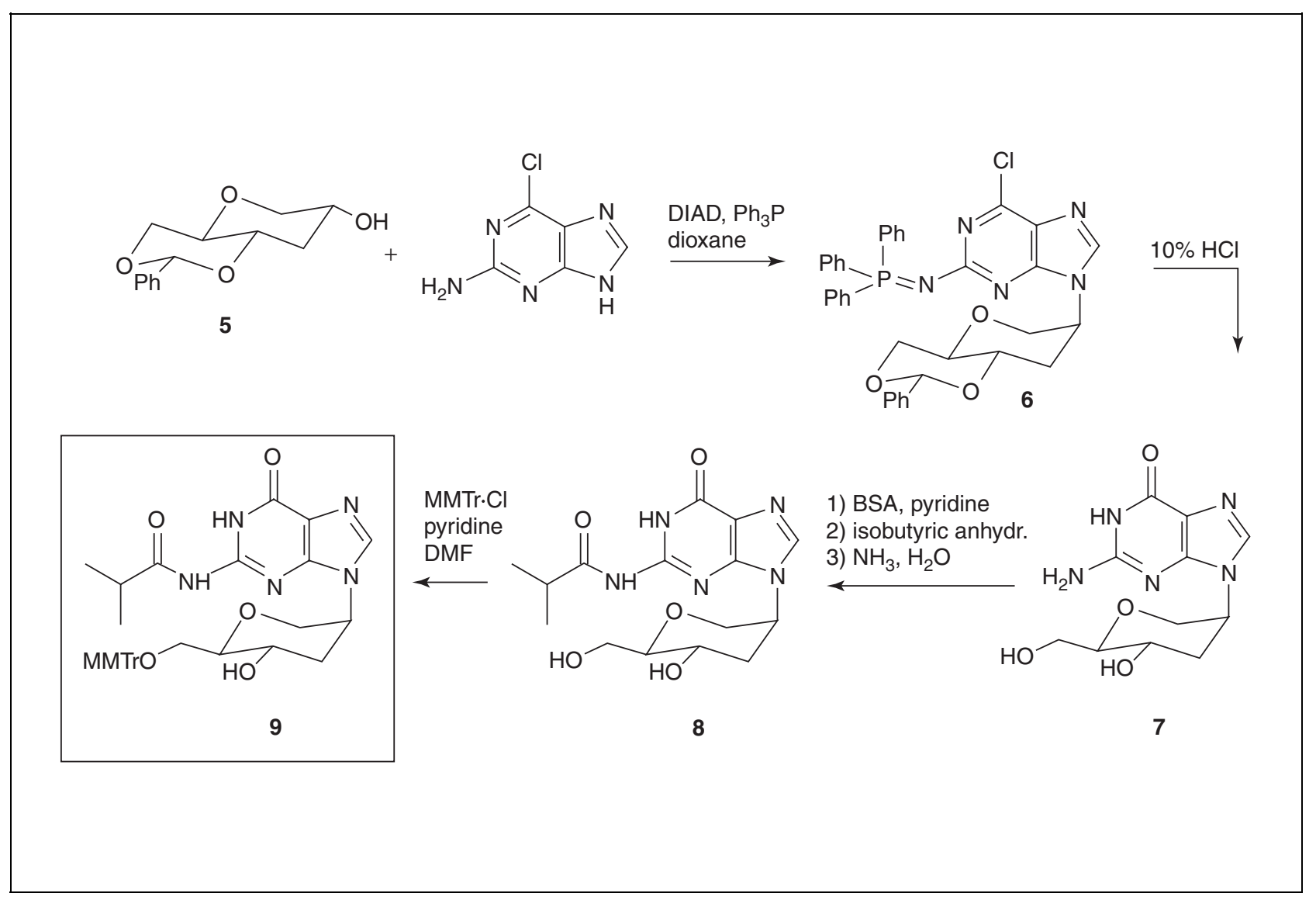

Figure 1.9.2 Preparation of protected $h \mathrm{G}$ (S.9). Abbreviations: BSA, bis(trimethylsilyl)acetamide; DIAD, diisopropyl azodicarboxylate; DMF, dimethylformamide; $\mathrm{MMTr}$. Cl, 4-monomethoxytrityl chloride; $\mathrm{Ph}_{3} \mathrm{P}$, triphenylphosphine.

Oil bath and magnetic stirrer

$5 \times 35-$ and $4 \times 25-$ cm chromatography columns

Additional reagents and equipment for TLC and column chromatography (see Basic Protocol 1 and Critical Parameters)

NOTE: The ${ }^{1} \mathrm{H}$ NMR and ${ }^{13} \mathrm{C}$ NMR spectra were determined with a $200 \mathrm{MHz}$ Varian Gemini spectrometer with tetramethylsilane as internal standard. Abbreviations: s, singlet; d, doublet; dd, double doublet; $\mathrm{t}$, triplet; br s, broad signal; $\mathrm{m}$, multiplet; ddd, double doublet of doublet; dm, double multiplet. Liquid secondary-ion (LSIMS) mass spectra were obtained using a KRATOS Concept ${ }^{1} \mathrm{H}$ mass spectrometer.

\section{Prepare S.6}

1. Suspend $6.78 \mathrm{~g}$ (40.0 mmol) 6-chloro- $9 H$-purine-2-amine, $4.72 \mathrm{~g}$ (20.0 mmol) S.5, and $13.12 \mathrm{~g}(50.0 \mathrm{mmol})$ triphenylphosphine in $200 \mathrm{~mL}$ dioxane. Carry out the reaction under a nitrogen gas atmosphere.

2. Add a solution of $9.84 \mathrm{~mL}$ ( $50.0 \mathrm{mmol})$ DIAD in $25 \mathrm{~mL}$ dioxane via a dropping funnel over a period of $200 \mathrm{~min}$.

3. Stir the mixture overnight at room temperature. Monitor by TLC (see Basic Protocol 1, step 3) using 3:2 (v/v) $n$-hexane/ethyl acetate. Visualize product using a UV lamp $(254 \mathrm{~nm})$.

4. Remove volatiles by evaporation under vacuum using rotary evaporator equipped with a vacuum pump and cooling trap.

Synthesis of Modified Nucleosides

\subsection{7}

Supplement 14 
5. Absorb the crude product on $15.0 \mathrm{~g}$ of 0.060 - to $0.200-\mathrm{nm}$ silica gel in a $5 \times 35-\mathrm{cm}$ chromatography column and elute with a gradient from $1.5 \mathrm{~L}$ of $3: 2(\mathrm{v} / \mathrm{v})$ to $1.5 \mathrm{~L}$ of $2: 3(\mathrm{v} / \mathrm{v}) n$-hexane/ethyl acetate. Collect fractions in $2 \times 18-\mathrm{cm}$ test tubes.

6. Draw a roster of the fraction collector on a TLC plate and apply a drop of each fraction to the corresponding field on the plate. Visualize product-containing fractions using a UV lamp $(254 \mathrm{~nm})$. Combine the product-containing fractions and remove solvents in vacuo on a rotary evaporator.

The resulting product, $1^{\prime}, 5^{\prime}$-anhydro-4',6'-O-benzylidene-2'-6-chloro-2[(triphenylphosphoranylidene)amino]-9H-purin-9-yl-2',3'-dideoxy-D-arabinohexitol (S.6) is obtained in $64 \%$ yield $(8.31 \mathrm{~g}, 12.8 \mathrm{mmol}) . \mathrm{mp}: 158^{\circ} \mathrm{C}$; LSIMS (thygly): $\mathrm{m} / \mathrm{z}: 648[\mathrm{M}+\mathrm{H}]^{+} ;{ }^{1} \mathrm{H} \mathrm{NMR}$ $\left(\mathrm{CDCl}_{3}\right): \delta=2.00\left(\mathrm{dt}, 1 \mathrm{H}, 3^{\prime} \mathrm{ax}-\mathrm{H}, \mathrm{J}=11.6 \mathrm{~Hz}, \mathrm{~J}=4.5 \mathrm{~Hz}\right), 2.29\left(\mathrm{br}, \mathrm{d}, 1 \mathrm{H}, 3^{\prime}-e q-\mathrm{H}, \mathrm{J}=\right.$ $13.0 \mathrm{~Hz}), 3.57\left(\mathrm{~m}, 2 \mathrm{H}, 5^{\prime}-\mathrm{H}, 4^{\prime}-\mathrm{H}\right), 3.76\left(t, 1 \mathrm{H}, 6^{\prime} \mathrm{ax}-\mathrm{H}, \mathrm{J}=9.7 \mathrm{~Hz}\right), 4.10\left(\mathrm{dd}, 1 \mathrm{H}, \mathrm{l}^{\prime} \mathrm{ax}-\mathrm{H}\right.$, $J=13.2 \mathrm{~Hz}, J=2.8 \mathrm{~Hz}), 4.36\left(\mathrm{~m}, 2 \mathrm{H}, \mathrm{l}^{\prime} \mathrm{eq}-\mathrm{H}, 6^{\prime} \mathrm{eq}-\mathrm{H}\right), 4.73\left(\mathrm{br}, \mathrm{s}, 1 \mathrm{H}, \mathrm{2}^{\prime}-\mathrm{H}\right), 5.49(\mathrm{~s}, 1 \mathrm{H}$, $\mathrm{PhCH}), 7.19-7.72$ (m, 21H, $\mathrm{H}$ aromat. $), 8.15(\mathrm{~s}, 1 \mathrm{H}, 8-\mathrm{H}) ;{ }^{13} \mathrm{C} \mathrm{NMR}\left(\mathrm{CDCl}_{3}\right): \delta=32.9$ $\left(C-3^{\prime}\right), 50.2\left(C-2^{\prime}\right), 68.9\left(C-6^{\prime}\right), 69.5\left(C-1^{\prime}\right), 73.9\left(C-4^{\prime}\right), 74.5\left(C-5^{\prime}\right), 101.8(P h C H), 122.8$ (C-5), 125.9 (,2,6-C Ph), 127.5-133.4 (C, CH Ar), 137.2 (C-6), 140.2 (C-8), $149.6(C-4)$, $158.7(C-2)$.

\section{Prepare 5.7}

7. Suspend $8.20 \mathrm{~g}(12.6 \mathrm{mmol}) \mathbf{S . 6}$ in $160 \mathrm{~mL}$ of $10 \% \mathrm{HCl}$. Heat the reaction mixture $2 \mathrm{hr}$ at $100^{\circ} \mathrm{C}$.

8. Cool to room temperature and wash the yellow-brown solution with $60 \mathrm{~mL}$ dichloromethane to remove benzaldehyde and triphenylphosphine.

9. Add a drop of phenolphthalein solution as an indicator and neutralize the aqueous layer with $120 \mathrm{~mL}$ of $4 \mathrm{~N}$ sodium hydroxide.

At $\mathrm{pH} 7$ the product begins to precipitate.

10. Concentrate the suspension in vacuo using a rotary evaporator.

11. Dissolve the obtained white product in $745 \mathrm{~mL}$ boiling water and filter hot.

12. Cool to room temperature and incubate the mixture overnight at $4{ }^{\circ} \mathrm{C}$.

13. Filter off the obtained crystals and dry over phosphorus pentoxide.

The resulting product, $1^{\prime}, 5^{\prime}$-anhydro-2', 3'-dideoxy-2'-(guanin-9-yl)-D-arabinohexitol (S.7) is obtained in $83 \%$ yield $(2.95 \mathrm{~g}, 10.5 \mathrm{mmol})$. $\mathrm{mp}:>300^{\circ} \mathrm{C} ; \mathrm{UV}\left(\mathrm{H}_{2} \mathrm{O}\right): \lambda_{\max } \varepsilon=253 \mathrm{~nm}$ (9100); LSIMS (thgly): $\mathrm{m} / \mathrm{z}: 282[\mathrm{M}+\mathrm{H}]^{+} ;{ }^{1} H \mathrm{HMR}\left(\mathrm{DMSO}-\mathrm{d}_{6}\right): \delta=1.80\left(\mathrm{~m}, 1 \mathrm{H}, 3^{\prime} \mathrm{ax}-\mathrm{H}\right)$, $2.17\left(\mathrm{br}, 1 \mathrm{H}, 3^{\prime} \mathrm{eq}-\mathrm{H}\right.$ ), 3.20-3.70 ( $\left.\mathrm{m}, 2 \mathrm{H}, 5^{\prime}-\mathrm{H}, 4^{\prime}-H, 6 \mathrm{~A}-\mathrm{H}\right), 3.79\left(\mathrm{dd}, 1 \mathrm{H}, \mathrm{l}^{\prime} \mathrm{ax}-\mathrm{H}, \mathrm{J}=12.5\right.$ $H z, J=2.2 \mathrm{~Hz}), 4.05-4.15\left(\mathrm{~m}, 2 \mathrm{H}, \mathrm{l}^{\prime} \mathrm{eq}-\mathrm{H}, 6 \mathrm{~B}-\mathrm{H}\right), 4.52\left(\mathrm{~s}, \mathrm{br}, 1 \mathrm{H}, \mathrm{2}^{\prime}-\mathrm{H}\right), 4.63\left(\mathrm{t}, 1 \mathrm{H}, \mathrm{6}^{\prime}-\mathrm{OH}\right.$, $J=6.0 \mathrm{~Hz}), 4.91\left(\mathrm{~d}, 1 \mathrm{H}, 4^{\prime}-\mathrm{OH}, \mathrm{J}=5.3 \mathrm{~Hz}\right), 6.46\left(\mathrm{br}, \mathrm{s}, 2 \mathrm{H}, \mathrm{NH}_{2}\right), 7.87(\mathrm{~s}, 1 \mathrm{H}, 8-\mathrm{H}) ;{ }^{13} \mathrm{C}$ NMR $\left(D M S O-d_{6}\right): \delta=36.3\left(3^{\prime}-C\right), 50.2\left(2^{\prime}-C\right), 61.0\left(6^{\prime}-C\right), 61.2\left(4^{\prime}-C\right), 68.4\left(1^{\prime}-C\right), 83.2$ $\left(5^{\prime}-C\right), 116.3(C-5), 136.9(C-8), 151.5(C-4), 154.1(C-2), 157.9(C-6)$.

\section{Prepare S.8}

14. Suspend $3.58 \mathrm{~g}$ (12.7 $\mathrm{mmol}) \mathbf{S . 7}$ in $160 \mathrm{~mL}$ pyridine.

15. Add $16.57 \mathrm{~mL}(63.7 \mathrm{mmol}) \mathrm{BSA}$ and reflux $8 \mathrm{hr}$.

16. Stir the dark-red solution overnight ( $16 \mathrm{hr})$ at room temperature.

17. Add $10.00 \mathrm{~g}(63.7 \mathrm{mmol})$ isobutyric anhydride and stir $24 \mathrm{hr}$.

18. Cool the mixture to $0^{\circ} \mathrm{C}$ and add $20 \mathrm{~mL}$ water.

Synthesis of 1,5-Anhydrohexitol 
20. Continue stirring $2 \mathrm{hr}$ at room temperature.

21. Evaporate the volatiles under vacuum and add $200 \mathrm{~mL}$ water.

22. Stir the suspension $10 \mathrm{~min}$, then filter off the precipitate. Wash once with $50 \mathrm{~mL}$ water followed by three washes of $100 \mathrm{~mL}$ of 1:1 (v/v) ethyl acetate/diethyl ether.

The resulting product, $1^{\prime}, 5^{\prime}$-anhydro-2',3'-dideoxy-2'-( $N^{2}$-isobutyrylguanin-9-yl)-D-arabinohexitol (S.8) is obtained in $90 \%$ yield $(3.74 \mathrm{~g}, 10.7 \mathrm{mmol}) . \mathrm{mp}: 258^{\circ} \mathrm{C}$; LSIMS (thgly): $m / z: 352[M+H]^{+} ;{ }^{l} H N M R\left(D M S O-d_{6}\right): \delta=1.13\left(d, 6 H, 2 \times \mathrm{CH}_{3}, J=7 \mathrm{~Hz}\right), 1.87(d t$, $\left.1 \mathrm{H}, 3^{\prime} \mathrm{ax}-\mathrm{H}, \mathrm{J}=12.3 \mathrm{~Hz}, 4.2 \mathrm{~Hz}\right), 2.23\left(\mathrm{br}, \mathrm{d}, 1 \mathrm{H}, 3^{\prime}\right.$ eq- $\left.\mathrm{H}, \mathrm{J}=12.8 \mathrm{~Hz}\right), 2.78(\mathrm{sept}, 1 \mathrm{H}, \mathrm{CH}$, $J=7 \mathrm{~Hz}), 3.18\left(\mathrm{~m}, 2 \mathrm{H}, 5^{\prime}-\mathrm{H}, 4^{\prime}-H\right), 3.45-3.75\left(\mathrm{~m}, 2 \mathrm{H}, 6^{\prime}-H\right), 3.85\left(\mathrm{dd}, 1 \mathrm{H}, \mathrm{l}^{\prime} a x-H, J=\right.$ $12.5 \mathrm{~Hz}, \mathrm{~J}=2.7 \mathrm{~Hz}), 4.17\left(\mathrm{~d}, 1 \mathrm{H}, \mathrm{l}^{\prime} \mathrm{eq}-\mathrm{H}, \mathrm{J}=12.4 \mathrm{~Hz}\right), 4.65$ (m, 2H, $\left.2^{\prime}-\mathrm{H}, 6^{\prime}-\mathrm{OH}\right), 5.01$ $\left(\mathrm{br}, \mathrm{s}, 1 \mathrm{H}, 4^{\prime}-\mathrm{OH}\right), 8.15(\mathrm{~s}, 1 \mathrm{H}, 8-\mathrm{H}), 10.2(\mathrm{br}, \mathrm{s}, 1 \mathrm{H}, \mathrm{NH}) ;{ }^{13} \mathrm{CNMR}\left(\mathrm{DMSO}-\mathrm{d}_{6}\right): \delta=19.0$ $\left(2 \times \mathrm{CH}_{3}\right), 34.8\left(\mathrm{C}-3^{\prime}\right), 36.2(\mathrm{CH}), 50.4\left(\mathrm{C}-2^{\prime}\right), 60.6\left(\mathrm{C}-6^{\prime}, \mathrm{C}-4^{\prime}\right), 68.0\left(\mathrm{C}-\mathrm{I}^{\prime}\right), 83.1\left(\mathrm{C}-5^{\prime}\right)$, $119.7(C-5), 138.6(C-8), 148.0(C-2), 148.7(C-4), 155.1(C-6), 180.2(H N C=O)$.

\section{Prepare S.9}

23. Suspend $4.03 \mathrm{~g}(11.5 \mathrm{mmol}) \mathbf{S . 8}$ in a mixture of $60 \mathrm{~mL} \mathrm{DMF}$ and $60 \mathrm{~mL}$ pyridine.

24. Heat the suspension to $120^{\circ} \mathrm{C}$ until a clear red-brown solution is obtained.

25. Cool to room temperature and add $4.6 \mathrm{~g}(14.92 \mathrm{mmol}, 1.3 \mathrm{eq}) \mathrm{MMTr} \cdot \mathrm{Cl}$.

26. Stir the reaction overnight at room temperature.

27. Monitor the reaction by TLC using 94:6 (v/v) dichloromethane/methanol and visualize the product using a UV lamp (the product has a higher $R_{\mathrm{f}}$ value than the starting material).

28. Quench the reaction with $200 \mathrm{~mL}$ saturated sodium bicarbonate solution.

29. Extract with $100 \mathrm{~mL}$ dichloromethane four times.

30. Dry the organic layer over sodium sulfate.

31. Remove the solvent and coevaporate with $10 \mathrm{~mL}$ toluene three times.

32. Purify by flash chromatography on a $4 \times 25-\mathrm{cm}$ column using a step gradient from $500 \mathrm{~mL}$ of $100 \%$ dichloromethane to 99:1 (1 L), 98:2 (1 L), and 97:3 (v/v) dichloromethane/methanol. Combine product-containing fractions and evaporate solvents in vacuo on a rotary evaporator.

The resulting product, $1^{\prime}, 5^{\prime}$-anhydro-2',3'-dideoxy-2'-( $N^{2}$-isobutyrylguanin-9-yl)-6'-Omonomethoxytrityl-D-arabinohexitol $(\boldsymbol{S . 9})$ is obtained in $93 \%$ yield $(6.65 \mathrm{~g}, 10.7 \mathrm{mmol})$. mp: $165^{\circ} \mathrm{C}$; LSIMS (thgly): $\mathrm{m} / \mathrm{z}: 646[\mathrm{M}+\mathrm{Na}]^{+} ;{ }^{l} \mathrm{H} \mathrm{NMR}\left(\mathrm{CDCl}_{3}\right): \delta=1.19(\mathrm{~d}, 6 \mathrm{H}, 2 x$ $\left.\left.\mathrm{CH}_{3}, \mathrm{~J}=6.8 \mathrm{~Hz}\right), 1.80\left(t, 1 \mathrm{H}, 3^{\prime} \mathrm{ax}-\mathrm{H}\right), \mathrm{J}=12.6 \mathrm{~Hz}\right), 2.20\left(\mathrm{~s}, 1 \mathrm{H}, 4^{\prime}-\mathrm{OH}\right), 2.32(\mathrm{br}, \mathrm{d}, 1 \mathrm{H}$, $3^{\prime}$ eq-H, $J=12.1 \mathrm{~Hz}$ ), $2.70(\mathrm{sept}, 1 \mathrm{H}, \mathrm{CH}, \mathrm{J}=6.9 \mathrm{~Hz}), 3.35-3.50\left(\mathrm{~m}, 4 \mathrm{H}, 5^{\prime}-\mathrm{H}, 4^{\prime}-\mathrm{H}, 6 \mathrm{~A}-\mathrm{H}\right.$, $6 B-H), 3.75\left(m, 4 H, l^{\prime} a x-H, O_{3}\right), 4.20\left(d, l H, l^{\prime} e q-H, J=13.4 \mathrm{~Hz}\right), 4.52(b r, s, l H$, $\left.2^{\prime}-H\right), 6.80(d, 2 H$, aromatic $H), 7.10-7.50(m, 12 H$, aromatic $H), 8.10(s, 1 H, 8-H), 9.66$ $(\mathrm{br}, \mathrm{s}, \mathrm{lH}, \mathrm{NH}) ;{ }^{13} \mathrm{C} \mathrm{NMR}\left(\mathrm{CDCl}_{3}\right): \delta=18.8\left(\mathrm{CH}_{3}\right), 18.9\left(\mathrm{CH}_{3}\right), 35.8\left(3^{\prime}-\mathrm{C}\right), 36.0(\mathrm{CH})$, $50.7\left(2^{\prime}-\mathrm{C}\right), 55.1\left(\mathrm{OCH}_{3}\right), 63.3\left(4^{\prime}-\mathrm{C}\right), 63.9\left(6^{\prime}-\mathrm{C}\right), 68.5\left(\mathrm{l}^{\prime}-\mathrm{C}\right), 81.1\left(5^{\prime}-\mathrm{C}\right), 86.7(\mathrm{O}-\mathrm{C}-\mathrm{Tr})$, $113.1\left(m^{\prime}-C, 2 C\right), 120.0(C-5), 126.9(p-C, 2 C), 127.8(o-C, 4 C), 128.3(m-C, 4 C), 130.2$ (o'-C, 2C), $135.2\left(i^{\prime} C\right), 138.6(C-8), 144.1(i C, 2 C), 147.8(C-2), 148.8(C-4), 156.0(C-6)$, $158.5\left(p^{\prime} C\right), 180.0(H N C=O)$.

Synthesis of 


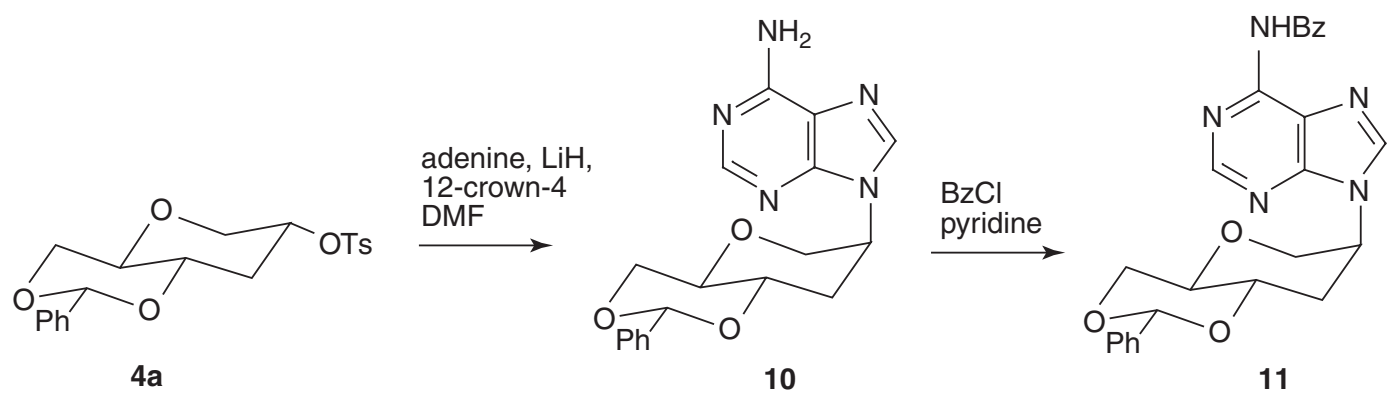

$4 a$

10

11

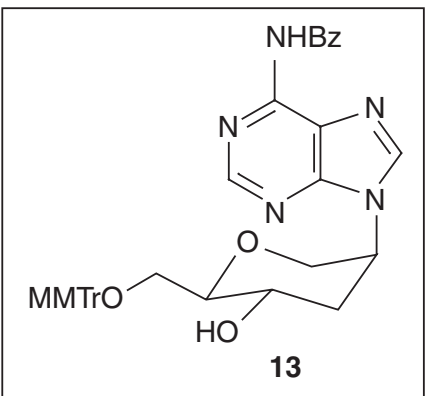

MMTr. Cl
pyridine

$\downarrow 80 \%$ HOAC

Figure 1.9.3 Preparation of protected $h \mathrm{~A}$ (S.13). Abbreviations: $\mathrm{BzCl}$, benzoyl chloride; DMF, dimethylformamide; HOAc, acetic acid; $\mathrm{MMTr} \cdot \mathrm{Cl}$, monomethoxytrityl chloride.

BASIC PROTOCOL 3

Synthesis of 1,5-Anhydrohexitol Building Blocks

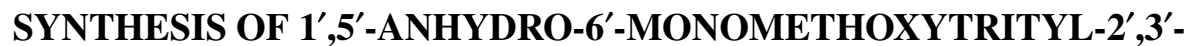
DIDEOXY-2' $-\left(N^{6}\right.$-BENZOYLADENIN-9-YL)-D-ARABINOHEXITOL

This protocol details the synthesis of the $h \mathrm{~A} 1,5$-anhydrohexitol building block $\mathbf{S . 1 3}$ from S.4a (see Fig. 1.9.3 and DeBouvere et al., 1997).

\section{Materials}

Adenine

Lithium hydride

12-Crown-4

Dimethylformamide (DMF; remove water by distillation with benzene followed by distillation under vacuum)

Nitrogen gas

1,5-Anhydro-4,6- $O$-benzylidene-3-deoxy-2- $O$-( $p$-tolylsulfonyl)-D-ribohexitol

(S.4a; see Basic Protocol 1)

$n$-Hexane

Ethyl acetate

Dichloromethane

Saturated sodium bicarbonate solution

Sodium sulfate

Methanol

Pyridine (reflux over potassium hydroxide overnight and distill)

Benzoyl chloride

$25 \%(\mathrm{v} / \mathrm{v})$ ammonia 
Toluene

$80 \%(\mathrm{v} / \mathrm{v})$ acetic acid

Diethyl ether: reflux overnight on sodium $\left(\mathrm{Na}, \mathrm{FeCl}_{2}, \mathrm{Et}_{2} \mathrm{O}\right)$ and distill

Pyridine (reflux over potassium hydroxide overnight and distill)

4-Monomethoxytrityl chloride (MMTr$\cdot \mathrm{Cl})$

Oil bath and magnetic stirrer

Rotary evaporator equipped with a vacuum pump and cooling trap

$3 \times 30-, 5 \times 40-$, and $4 \times 30-$ cm chromatography columns

Additional reagents and equipment for TLC and column chromatography (see

Basic Protocol 1 and Critical Parameters)

NOTE: The ${ }^{1} \mathrm{H}$ NMR and ${ }^{13} \mathrm{C}$ NMR spectra were determined with a $200 \mathrm{MHz}$ Varian Gemini spectrometer with tetramethylsilane as internal standard. Abbreviations: s, singlet; d, doublet; dd, double doublet; t, triplet; br s, broad signal; m, multiplet; ddd, double doublet of doublet; dm, double multiplet. Liquid secondary-ion (LSIMS) mass spectra were obtained using a KRATOS Concept ${ }^{1} \mathrm{H}$ mass spectrometer.

\section{Prepare S.10}

1. Suspend $1.35 \mathrm{~g}(10.0 \mathrm{mmol})$ adenine, $0.08 \mathrm{~g}(9.6 \mathrm{mmol})$ lithium hydride, and 0.32 $\mathrm{mL}$ of 12 -crown-4 (2.0 mmol) in $60 \mathrm{~mL}$ DMF. Carry out the reaction under a nitrogen gas atmosphere.

2. Heat the reaction mixture $1 \mathrm{hr}$ at $110^{\circ} \mathrm{C}$.

3. Add a solution of $1.95 \mathrm{~g}(5.0 \mathrm{mmol})$ of $\mathbf{S . 4 a}$ in $13 \mathrm{~mL}$ DMF with stirring.

4. Continue stirring at $110^{\circ} \mathrm{C}$ for $8 \mathrm{hr}$.

5. Test the reaction by TLC (see Basic Protocol 1, step 3) using 8:2 (v/v) hexane/ethyl acetate as solvent.

6. Cool the reaction mixture to room temperature and quench with $0.09 \mathrm{~mL}(5.0 \mathrm{mmol})$ water.

7. Concentrate the mixture under reduced pressure using a rotary evaporator with a vacuum pump.

8. Dissolve the residue in $100 \mathrm{~mL}$ dichloromethane and wash with $200 \mathrm{~mL}$ saturated sodium bicarbonate followed by two washes with $100 \mathrm{~mL}$ water.

9. Dry over sodium sulfate, filter, concentrate, and purify the residue by flash chromatography on a $3 \times 30-\mathrm{cm}$ column using a step gradient of 99:1 $(1 \mathrm{~L}), 98: 2(1 \mathrm{~L}), 97: 3$ $(1 \mathrm{~L})$, and 96:4 (v/v) dichloromethane/methanol. Combine product-containing fractions and evaporate solvent in vacuo on a rotary evaporator.

The resulting product, 2'-(adenin-9-yl)-1',5'-anhydro-4',6'-O-benzylidene-2', 3'-dideoxy$D$-arabinohexitol $(\mathbf{S . 1 0})$ is obtained in $82 \%$ yield $(1.45 \mathrm{~g}, 4.1 \mathrm{mmol}) . \mathrm{mp}: 227^{\circ} \mathrm{C} ; \mathrm{UV}$ $(\mathrm{MeOH}): \lambda_{\max }(\varepsilon)=262 \mathrm{~nm}(11300) ;$ EIMS: $\mathrm{m} / \mathrm{z}: 353[\mathrm{M}]^{+} ;{ }^{1} \mathrm{H}$ NMR $\left(\mathrm{DMSO}-d_{6}\right): \delta=$ $2.17\left(d t, 1 \mathrm{H}, \mathrm{H}-3^{\prime} a x, J=12 \mathrm{~Hz}, J=1.3 \mathrm{~Hz}, J=4 \mathrm{~Hz}\right), 2.46\left(\mathrm{~m}, 1 \mathrm{H}, \mathrm{H}-3^{\prime} e q\right), 3.53$ (ddd, $1 \mathrm{H}, \mathrm{H}-5^{\prime}, J=9 \mathrm{~Hz}, J=10 \mathrm{~Hz}, J=5 \mathrm{~Hz}$ ), $3.73\left(\mathrm{ddd}, 1 \mathrm{H}, \mathrm{H}-4^{\prime}, J=12.9 \mathrm{~Hz}, J=4 \mathrm{~Hz}\right.$ ), 3.80 $\left(t, 1 \mathrm{H}, \mathrm{H}-\mathrm{G}^{\prime} \mathrm{ax}, \mathrm{J}=10 \mathrm{~Hz}\right.$ ), $4.10\left(\mathrm{dd}, 1 \mathrm{H}, \mathrm{H}-\mathrm{l}^{\prime} \mathrm{ax}, \mathrm{J}=13 \mathrm{~Hz}, J=2.5 \mathrm{~Hz}\right), 4.22(\mathrm{dd}, 1 \mathrm{H}$, $\left.H-6^{\prime} e q, J=5 \mathrm{~Hz}\right), 4.44\left(\mathrm{~d}, 1 \mathrm{H}, \mathrm{H}-\mathrm{l}^{\prime} e q, J=13 \mathrm{~Hz}\right), 4.90\left(\mathrm{br}, \mathrm{s}, 1 \mathrm{H}, \mathrm{H}-2^{\prime}\right), 5.62(\mathrm{~s}, 1 \mathrm{H}$, PhCH), 7.30-7.40 ( $\mathrm{m}, 7 \mathrm{H}$, aromatic $\left.\mathrm{H}, \mathrm{NH}_{2}\right), 8.18(\mathrm{~s}, 1 \mathrm{H}, \mathrm{H}-2), 8.27(\mathrm{~s}, 1 \mathrm{H}, \mathrm{H}-8) ;{ }^{13} \mathrm{CNMR}$ $\left(D M S O-d_{6}\right): \delta=32.2\left(C-3^{\prime}\right), 50.5\left(C-2^{\prime}\right), 68.1,68.9\left(C-1^{\prime}, C-6^{\prime}\right), 73.4,73.8\left(C-4^{\prime}, C-5^{\prime}\right)$, $100.9(\mathrm{PhCH}), 118.6(\mathrm{C}-5), 126.2(2,6-\mathrm{C}), 128.1(3,5-\mathrm{C}), 128.9(4-\mathrm{C}), 137.8(1-\mathrm{C}), 139.3$ $(C-8), 149.5(C-4), 152.6(C-2), 156.2(C-6)$.

Synthesis of Modified Nucleosides

1.9.11 
Prepare S.11

10. Dissolve $5.90 \mathrm{~g}$ (16.7 $\mathrm{mmol}) \mathbf{S . 1 0}$ in $100 \mathrm{~mL}$ pyridine and coevaporate three times.

11. Add $160 \mathrm{~mL}$ pyridine and then cool to $0^{\circ} \mathrm{C}$.

12. Add $9.7 \mathrm{~mL}$ ( $83.57 \mathrm{mmol})$ benzoyl chloride.

13. Allow the mixture to come to room temperature and continue stirring overnight.

14. Cool the orange-brown solution in an ice-bath and add $18 \mathrm{~mL}$ water.

15. After $5 \mathrm{~min}$ add $35 \mathrm{~mL}$ of $25 \%$ ammonia and continue stirring $2 \mathrm{hr}$ at room temperature.

16. Remove the volatiles under reduced pressure and coevaporate three times with $20 \mathrm{~mL}$ toluene.

17. Dilute the resulting solid with $250 \mathrm{~mL}$ dichloromethane and wash with $200 \mathrm{~mL}$ saturated sodium bicarbonate solution.

18. Dry the organic layer over sodium sulfate, remove the solvent, and purify by column chromatography using a $5 \times 40-\mathrm{cm}$ silica gel column and a step gradient from $1.5 \mathrm{~L}$ of 2:8 (v/v) $n$-hexane/ethyl acetate to $1 \mathrm{~L}$ of $1: 9 n$-hexane/ethyl acetate, to $1 \mathrm{~L}$ of $100 \%$ ethyl acetate. Combine product-containing fractions and evaporate solvent.

The resulting product, $1^{\prime}, 5^{\prime}$-anhydro-2'-(N6-benzoyladenin-9-yl)-4',6'-O-benzylidene$2^{\prime}, 3^{\prime}$-dideoxy-D-arabinohexitol (S.11) is obtained in $94 \%$ yield $(7.20 \mathrm{~g}, 15.8 \mathrm{mmol})$. mp: $150^{\circ} \mathrm{C}$; LSIMS (thygly): $\mathrm{m} / z: 458[\mathrm{M}+\mathrm{H}]^{+} ;{ }^{l} \mathrm{H} \mathrm{NMR}\left(\mathrm{CDCl}_{3}\right): \delta=2.25\left(\mathrm{~m}, 1 \mathrm{H}, 3^{\prime} \mathrm{ax}-\mathrm{H}\right.$, $2.66\left(\mathrm{br}, \mathrm{d}, 1 \mathrm{H}, 3^{\prime} e q-H, J=12 \mathrm{~Hz}\right), 3.65\left(\mathrm{~m}, 2 \mathrm{H}, 5^{\prime}-H, 4^{\prime}-H\right), 3.78\left(t, 1 H, \sigma^{\prime} a x-H, J=9.8\right.$ $\mathrm{Hz}), 4.16\left(\mathrm{dd}, 1 \mathrm{H}, \mathrm{l}^{\prime} \mathrm{ax}-\mathrm{H}, \mathrm{J}=13.5 \mathrm{~Hz}, \mathrm{~J}=2.5 \mathrm{~Hz}\right), 4.38\left(\mathrm{dd}, 1 \mathrm{H}, 6^{\prime} \mathrm{eq}-\mathrm{H}, \mathrm{J}=10.2 \mathrm{~Hz}, \mathrm{~J}\right.$ $=3.6 \mathrm{~Hz}), 4.48\left(\mathrm{br}, \mathrm{d}, 1 \mathrm{H}, \mathrm{l}^{\prime} e q-H, J=13.3 \mathrm{~Hz}\right), 5.07\left(\mathrm{br}, \mathrm{s}, 1 \mathrm{H}, 2^{\prime}-\mathrm{H}, 5.49(\mathrm{~s}, 1 \mathrm{H}, \mathrm{PhCH})\right.$, $7.50(\mathrm{~m}, 8 \mathrm{H}$, aromatic $H), 8.04(\mathrm{~d}, 2 \mathrm{H}$, aromatic $H, J=6.7 \mathrm{~Hz}), 8.55(\mathrm{~s}, 1 \mathrm{H}, 8-\mathrm{H}), 8.77(\mathrm{~s}$, $1 \mathrm{H}, 2-\mathrm{H}), 9.30(\mathrm{~s}, 1 \mathrm{H}, \mathrm{NH}) ;{ }^{13} \mathrm{C} \mathrm{NMR}\left(\mathrm{CDCl}_{3}\right): \delta=33.1\left(\mathrm{C}-3^{\prime}\right), 51.0\left(\mathrm{C}-2^{\prime}\right), 68.8\left(\mathrm{C}-6^{\prime}\right)$, $69.4\left(C-1^{\prime}\right), 73.8\left(C-4^{\prime}\right), 74.5\left(C-5^{\prime}\right), 102.0(P h C H), 122.8(C-5), 125.9(2,6-C P h), 127.9$ (3,5C Ph), 128.2 (3,5-C Bz), 128.8 (2,6-C Bz), 129.1 (4-C Ph), 132.7 (4-C Bz), 133.5 (1-C Bz), 136.9 (1-C Ph), 142.0 (C-8), 149.6 (C-4), 152.0 (C-2), 152.5 (C-6), $164.7(H N C=O)$.

\section{Prepare S.12}

19. Dissolve $8.06 \mathrm{~g}$ (17.6 mmol) S.11 in $450 \mathrm{~mL}$ of $80 \%$ (v/v) acetic acid.

20. Heat the reaction mixture $6 \mathrm{hr}$ at $60^{\circ} \mathrm{C}$.

21. Evaporate the solvent under reduced pressure using a rotary evaporator with a vacuum pump and coevaporate with $25 \mathrm{~mL}$ toluene three times.

22. Dissolve the residue in a minimal volume of $1: 1(\mathrm{v} / \mathrm{v})$ dichloromethane/methanol.

23. Slowly add $500 \mathrm{~mL}$ diethyl ether while stirring.

24. Filter off the precipitate, wash with diethyl ether, and dry over phosphorous pentoxide.

The resulting product, $1^{\prime}, 5^{\prime}$-anhydro- $2^{\prime}$-( $N^{6}$-benzoyladenin-9-yl)-2', $3^{\prime}$-dideoxy-D-arabinohexitol (S.12) is obtained in $82 \%$ yield $(5.35 \mathrm{~g}, 14.5 \mathrm{mmol}) . \mathrm{mp}: 220^{\circ} \mathrm{C}$; LSIMS (thygly): $m / z: 370[M+H]^{+} ;{ }^{l} H$ NMR $\left(D M S O-d_{6}\right): \delta=1.97\left(d t, 1 H, 3^{\prime} a x-H, J=13 \mathrm{~Hz}, J=3.9 \mathrm{~Hz}\right)$, $2.36\left(\mathrm{br}, \mathrm{d}, 1 \mathrm{H}, 3^{\prime} e q-H, J=13.2 \mathrm{~Hz}\right), 3.23\left(\mathrm{~m}, 1 \mathrm{H}, 5^{\prime}-H\right), 3.50-3.80\left(\mathrm{~m}, 3 H, 6^{\prime}-H, 4^{\prime} H\right)$, $3.93\left(\mathrm{dd}, 1 \mathrm{H}, \mathrm{l}^{\prime} \mathrm{ax}-\mathrm{H}, \mathrm{J}=12.7 \mathrm{~Hz}, \mathrm{~J}=2.1 \mathrm{~Hz}\right), 4.30\left(\mathrm{br}, \mathrm{d}, 1 \mathrm{H}, \mathrm{l}^{\prime} \mathrm{eq}-\mathrm{H}, \mathrm{J}=12.5 \mathrm{~Hz}\right), 4.7$ $\left(t, 1 H, 6^{\prime}-O H, J=6.2 \mathrm{~Hz}\right), 4.98\left(\mathrm{br}, \mathrm{s}, 1 \mathrm{H}, 2^{\prime}-\mathrm{H}\right), 5.00\left(\mathrm{~d}, 1 \mathrm{H}, 4^{\prime}-\mathrm{OH}, \mathrm{J}=5.5 \mathrm{~Hz}\right), 7.58(\mathrm{~m}$, $3 H$, aromatic $H), 8.05(d, 2 H$, aromatic $H, J=6.9 H z), 8.62(s, 1 H, 8-H), 8.75(s, 1 H, 2-H)$, $11.18(\mathrm{~s}, 1 \mathrm{H}, \mathrm{NH}) ;{ }^{13} \mathrm{C} \mathrm{NMR}\left(\mathrm{DMSO}-\mathrm{d}_{6}\right): \delta=35.8\left(\mathrm{C}-3^{\prime}\right), 50.7\left(\mathrm{C}-2^{\prime}\right), 60.5\left(\mathrm{C}-6^{\prime}, \mathrm{C}-4^{\prime}\right)$, $67.9\left(C-1^{\prime}\right), 83.1\left(C-5^{\prime}\right) 125.1$ (C-5), 128.6 (2,3,5,6-C Bz), 132.5 (4-C Bz), 133.5 (1-C Bz), 143.5 (C-8), 150.2 (C-4), $151.5(C-2), 152.4(C-6), 165.7(H N C=O)$. 
Prepare S.13

25. Dissolve $5.35 \mathrm{~g}$ (14.5 mmol) $\mathbf{S . 1 2}$ in $100 \mathrm{~mL}$ pyridine and coevaporate three times.

26. Add $340 \mathrm{~mL}$ pyridine and dissolve.

27. Add $7.85 \mathrm{~g}(24.6 \mathrm{mmol}) \mathrm{MMTr} \cdot \mathrm{Cl}$.

28. Stir the reaction 2 days at room temperature.

29. Monitor by TLC using 94:6 (v/v) dichloromethane/methanol (the product has a higher $R_{\mathrm{f}}$ value than the starting material).

30. Quench the reaction with $300 \mathrm{~mL}$ saturated sodium bicarbonate solution.

31. Extract with $400 \mathrm{~mL}$ dichloromethane twice.

32. Dry the organic layer over sodium sulfate, evaporate the solvent, and then coevaporate with $25 \mathrm{~mL}$ toluene three times.

33. Purify the residue by flash chromatography on a $4 \times 30-\mathrm{cm}$ column using a step gradient from 1.5 L of 100\% dichloromethane to 99:1 (1 L), 98:2 (1 L), and 97:3 $(\mathrm{v} / \mathrm{v})$ dichloromethane/methanol. Combine product-containing fractions and evaporate the solvent.

The resulting product, $1^{\prime}, 5^{\prime}$-anhydro-6'-monomethoxytrityl-2',3'-dideoxy-2'-( $N^{6}$-benzoyladenin-9-yl)-D-arabinohexitol (S.13) is obtained in $84 \%$ yield $(7.79 \mathrm{~g}, 12.2 \mathrm{mmol}) . \mathrm{mp}$ : $125^{\circ} \mathrm{C}$; LSIMS (thygly/NaOAc): $\mathrm{m} / \mathrm{z}: 664[\mathrm{M}+\mathrm{Na}]^{+} ;{ }^{1} \mathrm{H} \mathrm{NMR}\left(\mathrm{CDCl}_{3}\right): \delta=1.98(\mathrm{dt}, \mathrm{lH}$, $\left.3^{\prime} \mathrm{ax}-\mathrm{H}, \mathrm{J}=11.6 \mathrm{~Hz}, J=4.4 \mathrm{~Hz}\right), 2.54\left(\mathrm{br}, \mathrm{d}, 1 \mathrm{H}, 3^{\prime} \mathrm{eq}-\mathrm{H}, \mathrm{J}=12.6 \mathrm{~Hz}\right), 2.88(\mathrm{br}, \mathrm{s}, 1 \mathrm{H}$, $\left.4^{\prime}-\mathrm{OH}\right), 3.47\left(\mathrm{~m}, 3 \mathrm{H}, 5^{\prime}-\mathrm{H}, 4^{\prime}-\mathrm{H}, 6 \mathrm{~A}-\mathrm{H}\right), 3.77\left(\mathrm{br}, \mathrm{s}, 4 \mathrm{H}, 6 \mathrm{~B}-\mathrm{H}, \mathrm{OCH}_{3}\right), 3.96\left(\mathrm{dd}, 1 \mathrm{H}, \mathrm{I}^{\prime} \mathrm{ax}-\mathrm{H}\right.$, $J=12.9 \mathrm{~Hz}, J=2.5 \mathrm{~Hz}), 4.34\left(\mathrm{br}, \mathrm{d}, 1 \mathrm{H}, \mathrm{l}^{\prime} \mathrm{eq}-\mathrm{H}, \mathrm{J}=12.8 \mathrm{~Hz}\right), 4.98\left(\mathrm{br}, \mathrm{s}, 1 \mathrm{H}, \mathrm{2}^{\prime}-\mathrm{H}\right), 6.84$ $(d, 2 H$, aromatic $H, J=8.9 \mathrm{~Hz}), 7.38(\mathrm{~m}, 15 \mathrm{H}$, aromatic $H), 8.02(\mathrm{~d}, 2 \mathrm{H}$, aromatic $H, J=$ $8.3 \mathrm{~Hz}), 8.54(\mathrm{~s}, 1 \mathrm{H}, 8-\mathrm{H}), 8.77(\mathrm{~s}, 1 \mathrm{H}, 2-\mathrm{H}), 9.30(\mathrm{br}, \mathrm{s}, 1 \mathrm{H}, \mathrm{NH}) ;{ }^{13} \mathrm{C} \mathrm{NMR}\left(\mathrm{CDCl}_{3}\right): \delta=$ $35.7\left(C-3^{\prime}\right), 50.6\left(C-2^{\prime}\right), 55.2\left(\mathrm{OCH}_{3}\right), 64.3\left(C-4^{\prime}, C-6^{\prime}\right), 69.0\left(C-1^{\prime}\right), 80.6\left(C-5^{\prime}\right), 87.2$ (O-C-MMTr), 113.1 ( $\left.3^{\prime} 5^{\prime} C \mathrm{MMTr}\right), 120.0(\mathrm{C}-5), 127.1$ (4C MMTr $\left.2 x\right), 127.9$ (3,5C Bz), 128.0 (2,6C MMTr, 2×), 128.2 (3,5C MMTr, 2x), 128.8 (2,6C Bz), $130.2\left(2^{\prime} 6^{\prime} C \mathrm{MMTr}\right)$, 132.7 (4C Bz), 133.7 (lC Bz), 134.9(1'C MMTr), 142.4 (C-8), 143.8(1C MMTr, 2×), 149.5 $(C-4), 152.5(C-2, C-6), 158.7(H N C=O)$.

\section{SYNTHESIS OF 1',5'-ANHYDRO-6'-O-MONOMETHOXYTRITYL-2' 1' $^{\prime}$ - DIDEOXY-2' -(THYMIN-1-YL)-D-ARABINOHEXITOL}

This protocol details the synthesis of the $h \mathrm{~T} 1,5$-anhydrohexitol building block $\mathbf{S . 1 6}$ from S.5 (see Fig. 1.9.4 and DeBouvere et al., 1997).

\section{Materials}

$N^{3}$-Benzoylthymine

1,5-Anhydro-4,6-O-benzylidene-3-deoxy-D-glucitol (S.5; see Basic Protocol 1)

Triphenylphosphine

Tetrahydrofuran (THF; reflux overnight on lithium aluminum hydride and distill)

Nitrogen gas

Diethyl azodicarboxylate (DEAD)

$n$-Hexane

Ethyl acetate

Saturated ammonia in methanol

Dichloromethane

Toluene

$80 \%(\mathrm{v} / \mathrm{v})$ acetic acid

Methanol 


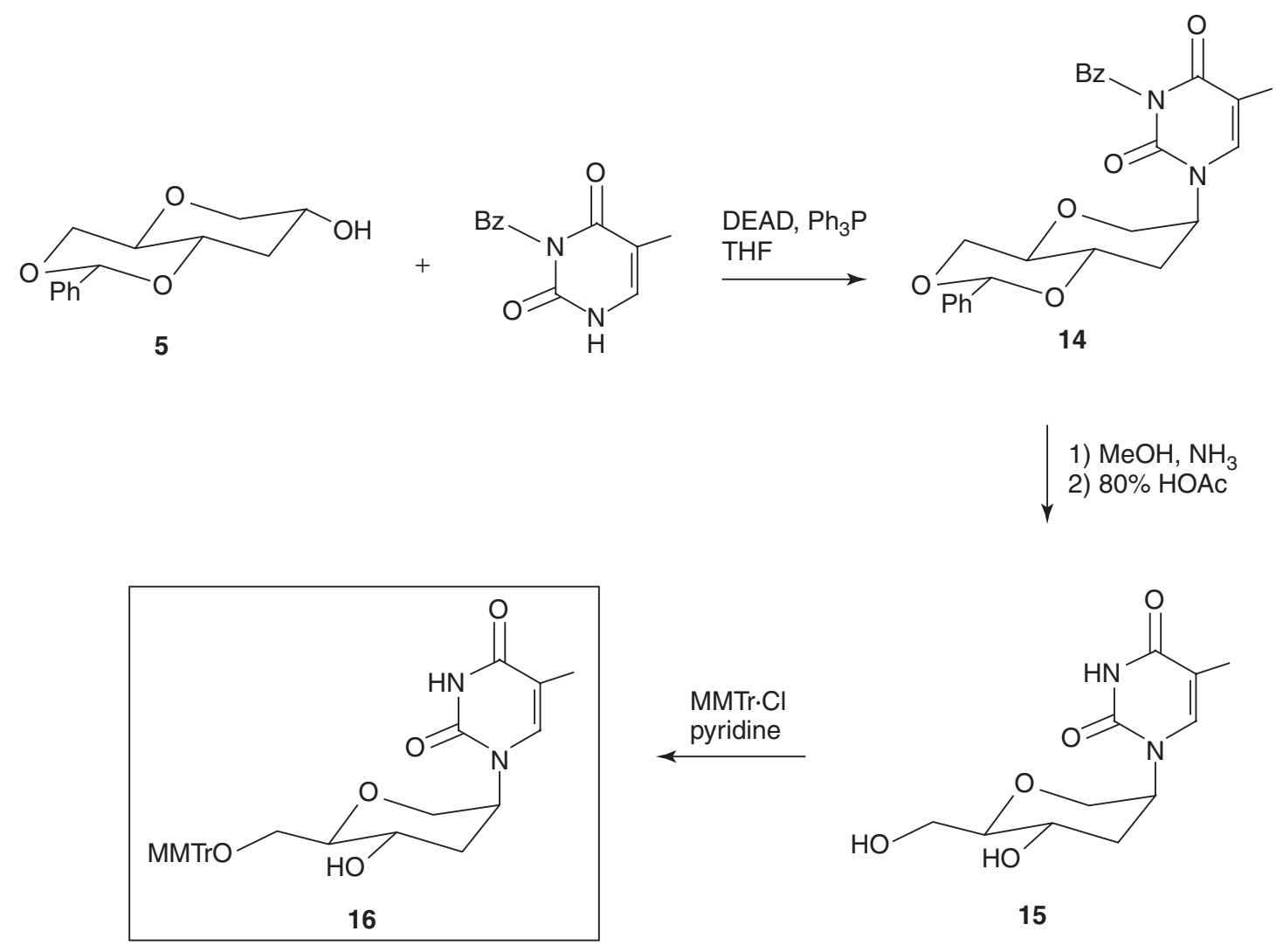

Figure 1.9.4 Preparation of protected $h \mathrm{~T}$ (S.16). Abbreviations: DEAD, diethyl azodicarboxylate; THF, tetrahydrofuran; $\mathrm{Ph}_{3} \mathrm{P}$, triphenylphosphine; HOAc, acetic acid; MMTr. $\mathrm{Cl}$, monomethoxytrityl chloride.

Synthesis of

Pyridine (reflux over potassium hydroxide overnight and distill)

Monomethoxytrityl chloride $(\mathrm{MMTr} \cdot \mathrm{Cl})$

Saturated sodium bicarbonate solution

Sodium sulfate

Oil bath and magnetic stirrer

Dropping funnel

Rotary evaporator equipped with a vacuum pump and cooling trap

$3 \times 35-$ and $4 \times 35-\mathrm{cm}$ chromatography columns

Additional reagents and equipment for TLC and column chromatography (see Basic Protocol 1 and Critical Parameters)

NOTE: The ${ }^{1} \mathrm{H}$ NMR and ${ }^{13} \mathrm{C}$ NMR spectra were determined with a 200-MHz Varian Gemini spectrometer with tetramethylsilane as internal standard. Liquid secondary-ion (LSIMS) mass spectra were obtained using a KRATOS Concept ${ }^{1} \mathrm{H}$ mass spectrometer. Abbreviations: s, singlet; d, doublet; dd, double doublet; t, triplet; br s, broad signal; m, multiplet; ddd, double doublet of doublet; $\mathrm{dm}$, double multiplet. 
1. Dissolve $2.40 \mathrm{~g}$ (10.5 mmol) $N^{3}$-benzoylthymine, $1.23 \mathrm{~g}(5.2 \mathrm{mmol}) \mathrm{S.5}$, and $3.43 \mathrm{~g}$ (13.1 mmol) triphenylphosphine in $100 \mathrm{~mL}$ THF. Carry out the reaction under a nitrogen atmosphere.

2. Add a solution of $2.06 \mathrm{~mL}$ (13.1 mmol) DEAD in $15 \mathrm{~mL}$ THF via a dropping funnel over a period of $60 \mathrm{~min}$.

3. Stir the mixture at room temperature overnight.

4. Remove the volatiles under vacuum using a rotary evaporator with a vacuum pump.

5. Absorb the crude product on $20 \mathrm{~g}$ of 0.060 - to 0.200 -nm silica gel and purify by flash chromatography in a $3 \times 35-\mathrm{cm}$ column using a step gradient of $1 \mathrm{~L}$ each of $8: 2,7: 3$, and 6:4 (v/v) $n$-hexane/ethyl acetate. Combine product-containing fractions and evaporate solvent in vacuo on a rotary evaporator.

The resulting product, $1^{\prime}, 5^{\prime}$-anhydro-2'-(N3-benzoylthymin-1-yl)-4', $6^{\prime}$-O-benzylidene$2^{\prime}, 3^{\prime}$-dideoxy-D-arabinohexitol (S.14) is obtained in $80 \%$ yield $(1.88 \mathrm{~g}, 4.2 \mathrm{mmol}) . \mathrm{mp}$ : $200^{\circ} \mathrm{C}$; LSIMS (thygly): $\mathrm{m} / \mathrm{z}: 449\left[\mathrm{M}+\mathrm{H}^{+}{ }^{+}{ }^{l}{ }^{\mathrm{H} \mathrm{NMR}}\left(\mathrm{CDCl}_{3}\right): \delta=2.02\left(\mathrm{~s}, 3 \mathrm{H}, \mathrm{CH}_{3}\right), 2.07\right.$ ( $\left.m, 1 H, 3^{\prime} a x-H\right), 2.47\left(d, b r, 1 H, 3^{\prime} e q-H, J=13.9 H z\right), 3.53\left(d t, 1 H, 5^{\prime}-H, J=9.6 H z, J=\right.$ $4.7 \mathrm{~Hz}), 3.8\left(\mathrm{~m}, 1 \mathrm{H}, 4^{\prime}-H\right), 3.80\left(t, 1 \mathrm{H}, \sigma^{\prime} \mathrm{ax}-\mathrm{H}, \mathrm{J}=10.2 \mathrm{~Hz}\right), 4.02\left(\mathrm{dd}, 1 \mathrm{H}, \mathrm{l}^{\prime} \mathrm{ax}-\mathrm{H}, \mathrm{J}=\right.$ $13.7 \mathrm{~Hz}, \mathrm{~J}=3.5 \mathrm{~Hz}), 4.28\left(\mathrm{~d}, \mathrm{br}, 1 \mathrm{H}, \mathrm{l}^{\prime} \mathrm{eq}-\mathrm{H}, \mathrm{J}=13.9 \mathrm{~Hz}\right), 4.37\left(\mathrm{dd}, 1 \mathrm{H}, \mathrm{\sigma}^{\prime}\right.$ eq- $\mathrm{H}, \mathrm{J}=10.5$ $\mathrm{Hz}, \mathrm{J}=4.7 \mathrm{~Hz}), 4.73\left(\mathrm{~s}, \mathrm{br}, 1 \mathrm{H}, 2^{\prime}-\mathrm{H}\right), 5.64(\mathrm{~s}, 1 \mathrm{H}, \mathrm{PhCH}), 7.30-7.90(\mathrm{~m}, 8 \mathrm{H}$, aromatic $\mathrm{H})$, $7.95(s,+d, 3 H, 6-H+$ aromatic $2 H) ;{ }^{13} C N M R\left(\mathrm{CDCl}_{3}\right): \delta=32.9\left(\mathrm{C}-3^{\prime}\right), 51.7\left(\mathrm{C}-2^{\prime}\right)$, $68.8\left(C-6^{\prime}, C-1^{\prime}\right), 73.6\left(C-4^{\prime}\right), 74.2\left(C-5^{\prime}\right), 102.0(P h C H), 110.7(C-5), 126.0(2,6 C P h)$, $128.3(4 C \mathrm{Ph}), 129.1$ (2,3,56C Bz), 130.4 (3,5 C Ph), 131.5 (1C Bz), 135.0 (4C Bz), 137.0 (1C Ph), $137.7(C-6), 149.7(C-2), 162.6(C-4), 168.9(H N C=O)$.

\section{Prepare S.15}

6. Dissolve $1.83 \mathrm{~g}$ (4.1 mmol) $\mathbf{S . 1 4}$ in $100 \mathrm{~mL}$ saturated ammonia in methanol.

7. Once a precipitate has formed, add $50 \mathrm{~mL}$ dichloromethane and continue stirring 90 min at room temperature.

8. Check the reaction by TLC (see Basic Protocol 1, step 3) using 1:1 (v/v) $n$-hexane/ethyl acetate (the starting material has the higher $R_{\mathrm{f}}$ value).

9. Evaporate the volatiles and coevaporate three times with $15 \mathrm{~mL}$ toluene.

10. Dissolve the crude product ( $1.8 \mathrm{~g}$ ) in $75 \mathrm{~mL}$ of $80 \%$ (v/v) acetic acid.

11. Heat the reaction mixture $3 \mathrm{hr}$ at $60^{\circ} \mathrm{C}$.

12. Evaporate the volatiles on a rotary evaporator and coevaporate three times with 15 $\mathrm{mL}$ toluene.

13. Purify the residue by column chromatography using a $3 \times 25-\mathrm{cm}$ column and a gradient from $1 \mathrm{~L}$ of 95:5 to $1 \mathrm{~L}$ of 93:7 (v/v) dichloromethane/methanol. Combine product-containing fractions and evaporate the solvent.

The resulting product, $1^{\prime}, 5^{\prime}$-anhydro-2',3'-dideoxy-2'-(thymin-1-yl)-D-arabinohexitol (S.15) is obtained in $82 \%$ yield $(0.86 \mathrm{~g}, 3.4 \mathrm{mmol}) . \mathrm{mp}: 170^{\circ} \mathrm{C} ; \mathrm{UV}(\mathrm{MeOH}): \lambda_{\max }(\varepsilon)=$ $272 \mathrm{~nm}$ (9500); LSIMS (thygly): $\mathrm{m} / \mathrm{z}: 357[\mathrm{M}+\mathrm{H}]^{+} ;{ }^{1} \mathrm{H} N \mathrm{NM}\left(\mathrm{DMSO}-d_{6}\right): \delta=1.75 \mathrm{~m}, 1 \mathrm{H}$, $\left.3^{\prime} \mathrm{ax}-\mathrm{H}\right), 1.76\left(\mathrm{~s}, 3 \mathrm{H}, \mathrm{CH}_{3}\right), 2.08\left(\mathrm{~d}, \mathrm{br}, 1 \mathrm{H}, 3^{\prime} \mathrm{eq}-\mathrm{H}, \mathrm{J}=13.8 \mathrm{~Hz}\right), 3.13\left(\mathrm{~m}, \mathrm{lH}, 5^{\prime}-\mathrm{H}\right), 3.35$ $\left(\mathrm{m}, 1 \mathrm{H}, 4^{\prime}-\mathrm{H}\right), 3.60\left(\mathrm{~m}, 2 \mathrm{H}, 6^{\prime}-\mathrm{H}\right), 3.73\left(\mathrm{dd}, 1 \mathrm{H}, \mathrm{l}^{\prime} \mathrm{ax}-\mathrm{H}, \mathrm{J}=12.9 \mathrm{~Hz}, \mathrm{~J}=3.4 \mathrm{~Hz}\right), 3.99$ (d,br, $\left.1 \mathrm{H}, \mathrm{l}^{\prime} \mathrm{eq}-\mathrm{H}, \mathrm{J}=12.8 \mathrm{~Hz}\right), 4.50\left(\mathrm{~s} b r, 1 \mathrm{H}, 2^{\prime}-\mathrm{H}\right), 4.65\left(\mathrm{~s}, \mathrm{br}, 1 \mathrm{H}, 6^{\prime}-\mathrm{OH}\right), 4.91(\mathrm{~s}, \mathrm{br}$, $\left.1 \mathrm{H}, 4^{\prime}-\mathrm{OH}\right), 7.88(\mathrm{~s}, 1 \mathrm{H}, 6-\mathrm{H}), 11.25(\mathrm{~s}, 1 \mathrm{H}, 3-\mathrm{H}) ;{ }^{13} \mathrm{C} \mathrm{NMR}\left(\mathrm{DMSO}-\mathrm{d}_{6}\right): \delta=12.5\left(\mathrm{CH}_{3}\right)$, $35.2\left(C-3^{\prime}\right), 50.1\left(C-2^{\prime}\right), 60.3\left(C^{\prime}-6^{\prime}\right), 60.7\left(C-4^{\prime}\right), 67.0\left(C-1^{\prime}\right), 82.4\left(C-5^{\prime}\right), 108.4(C-5)$, $139.0(C-6), 151.0(C-2), 163.9(C-4)$. 
Prepare S.16

14. Dissolve $2.77 \mathrm{~g}$ (10.8 mmol) $\mathbf{S . 1 5}$ in $40 \mathrm{~mL}$ pyridine and coevaporate three times.

15. Dissolve in $100 \mathrm{~mL}$ pyridine.

16. Add $5.85 \mathrm{~g}(18.4 \mathrm{mmol}) \mathrm{MMTr} \cdot \mathrm{Cl}$.

17. Stir the reaction at room temperature overnight.

18. Check by TLC using 9:1 (v/v) dichloromethane/methanol as the solvent (the reaction mixture has the higher $R_{\mathrm{f}}$ value).

19. Quench the reaction with $200 \mathrm{~mL}$ saturated sodium bicarbonate solution.

20. Extract twice with $200 \mathrm{~mL}$ dichloromethane.

21. Dry the organic layer over sodium sulfate, evaporate the solvent, and coevaporate with $15 \mathrm{~mL}$ toluene three times.

22. Purify the residue by column chromatography on a $4 \times 35-\mathrm{cm}$ column using a step gradient from $500 \mathrm{~mL}$ of $100 \%$ dichloromethane to $1 \mathrm{~L}$ each of 99:1, 98:2, and 95:5 $(\mathrm{v} / \mathrm{v})$ dichloromethane/methanol. Combine product-containing fractions and evaporate solvents.

The resulting product, $1^{\prime}, 5^{\prime}$-anhydro-6'-O-monomethoxytrityl-2',3'-dideoxy-2'-(thymin-1$y l$ )-D-arabinohexitol (S.16) is obtained in $86 \%$ yield $(4.90 \mathrm{~g}, 9.3 \mathrm{mmol})$. $\mathrm{mp}: 125^{\circ} \mathrm{C}$; LSIMS (thygly / NaOAc): $\mathrm{m} / z: 551[\mathrm{M}+\mathrm{Na}]^{+} ;{ }^{1} \mathrm{H} \mathrm{NMR}\left(\mathrm{CDCl}_{3}\right): \delta=1.85\left(\mathrm{~m}, \mathrm{lH}, 3^{\prime} \mathrm{ax}-\mathrm{H}\right), 1.86$ $\left(\mathrm{s}, 3 \mathrm{H}, \mathrm{CH}_{3}\right), 2.28\left(\mathrm{~d}, 1 \mathrm{H}, 4^{\prime}-\mathrm{OH}, \mathrm{J}=3.8 \mathrm{~Hz}\right), 2.38\left(\mathrm{~d}, \mathrm{br}, 1 \mathrm{H}, 3^{\prime} \mathrm{eq}-\mathrm{H}, \mathrm{J}=14 \mathrm{~Hz}\right), 3.31(\mathrm{~m}$, $\left.1 \mathrm{H}, 5^{\prime}-\mathrm{H}\right), 3.45\left(\mathrm{~m}, 2 \mathrm{H}, 4^{\prime} \mathrm{H}, 6^{\prime} \mathrm{ax}-\mathrm{H}\right), 3.78\left(\mathrm{~s}, 3 \mathrm{H}, \mathrm{OCH}_{3}\right), 3.81\left(\mathrm{dd}, 1 \mathrm{H}, \mathrm{l}^{\prime} \mathrm{ax}-\mathrm{H}, \mathrm{J}=13 \mathrm{~Hz}\right.$, $J=3.4 \mathrm{~Hz}), 3.98\left(\mathrm{~m}, 1 \mathrm{H}, 6^{\prime} \mathrm{eq}-\mathrm{H}\right), 4.18\left(\mathrm{~d}, \mathrm{br}, 1 \mathrm{H}, \mathrm{l}^{\prime} \mathrm{eq}-\mathrm{H}, \mathrm{J}=13.2 \mathrm{~Hz}\right), 4.67(\mathrm{~s}, \mathrm{br}, \mathrm{lH}$, $\left.2^{\prime}-H\right), 6.83(\mathrm{~d}, 2 \mathrm{H}$, aromatic $\mathrm{H}, \mathrm{J}=8.7 \mathrm{~Hz}), 7.20-7.50(\mathrm{~m}, 12 \mathrm{H}$, aromatic $\mathrm{H}), 8.00(\mathrm{~s}, 1 \mathrm{H}$, 6-H), $9.20(\mathrm{~s}, \mathrm{br}, \mathrm{lH}, \mathrm{NH}) ;{ }^{13} \mathrm{C} \mathrm{NMR}\left(\mathrm{CDCl}_{3}\right): \delta=12.8\left(\mathrm{CH}_{3}\right), 35.5\left(\mathrm{C}-3^{\prime}\right), 51.0\left(\mathrm{C}-2^{\prime}\right)$, $55.2\left(\mathrm{OCH}_{3}\right), 63.1\left(\mathrm{C}-4^{\prime}\right), 63.3\left(\mathrm{C}-6^{\prime}\right), 68.5\left(\mathrm{C}-\mathrm{I}^{\prime}\right), 80.9\left(\mathrm{C}^{\prime} 5^{\prime}\right), 86.8(\mathrm{OC} \mathrm{MMTr}), 110.4$ (C-5), $113.2\left(3^{\prime} 5^{\prime} C \mathrm{MMTr}\right), 127.1$ (4 MMTr, 2×), $128.0(2,6 \mathrm{C} M M T r, 2 \times), 128.2(3,5 \mathrm{C}$ MMTr, 2×), 130.2 (2'6'C MMtr), 135.0 (1'C MMTr), $138.6(C-6), 143.9$ (1C MMTr, 2×), $151.0(C-2), 158.7\left(4^{\prime} C M M T r\right), 163.8(C-4)$.

BASIC PROTOCOL 5

Synthesis of 1,5-Anhydrohexitol Building Blocks

1.9.16

SYNTHESIS OF 1',5'-ANHYDRO-2' $\mathbf{1}^{\prime}\left(N^{4}\right.$-BENZOYLCYTOSIN-1-YL)-2 $\mathbf{2}^{\prime}, \mathbf{3}^{\prime}$ DIDEOXY-6' -MONOMETHYOXYTRITYL-D-ARABINOHEXITOL

This protocol details the synthesis of the $h \mathrm{C} 1,5$-anhydrohexitol building block $\mathbf{S . 2 0}$ from S.5 (see Fig. 1.9.5 and DeBouvere et al., 1997).

\section{Materials}

$N^{4}$-Benzoyluracil

1,5-Anhydro-4,6-O-benzylidene-3-deoxy-D-glucitol (S.5; see Basic Protocol 1)

Triphenylphosphine

Dioxane (reflux overnight on lithium aluminum hydride and distill)

Nitrogen gas

Diethyl azodicarboxylate (DEAD)

Saturated ammonia in methanol

Dichloromethane

Methanol

Toluene

Triazole

Pyridine (reflux overnight over potassium hydroxide and distill)

Phosphoroxy chloride

$25 \%$ ammonia 


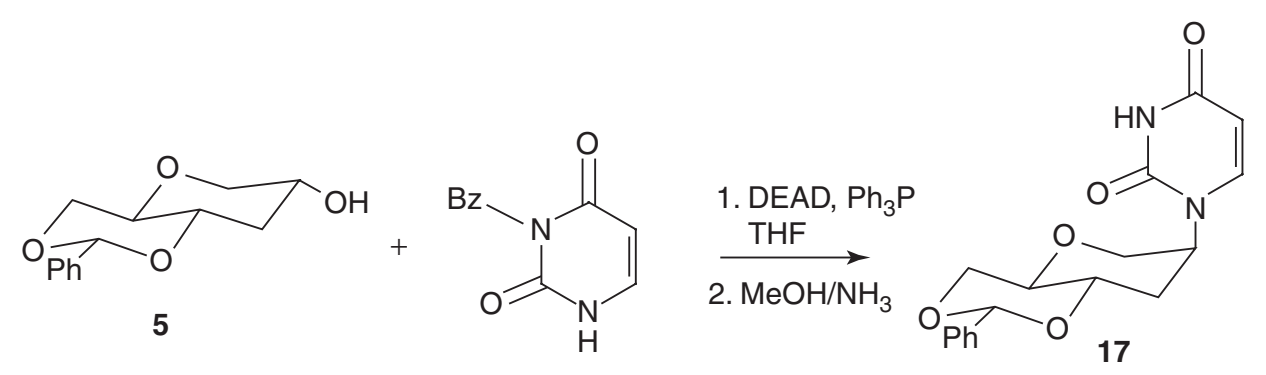

1. triazole/ $/ \mathrm{POCl}_{3} \downarrow$
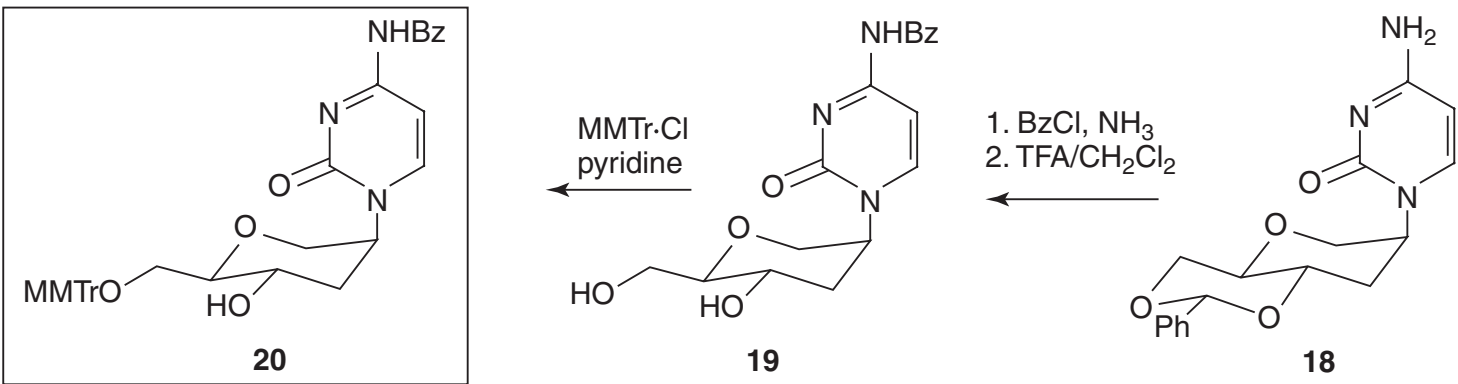

Figure 1.9.5 Preparation of protected $h \mathrm{C}$ (S.20). Abbreviations: BzCl, benzoylchloride; DEAD, diethyl azodicarboxylate; MMTr.Cl, 4-monomethoxy chloride; $\mathrm{POCl}_{3}$, phosphoroxy chloride; TFA, trifluoroacetic acid.

Sodium sulfate

Benzoyl chloride

Saturated sodium bicarbonate solution

Trifluoroacetic acid (TFA)

Diethyl ether: reflux overnight on sodium $\left(\mathrm{Na}, \mathrm{FeCl}_{2}, \mathrm{Et}_{2} \mathrm{O}\right)$ and distill

Monomethoxytrityl chloride (MMTr. $\mathrm{Cl})$

Dropping funnel

Rotary evaporator equipped with a vacuum pump and cooling trap

$4 \times 30$ - and $3 \times 35-\mathrm{cm}$ chromatography columns

Oil bath and magnetic stirrer

Drying tube

Additional reagents and equipment for TLC and column chromatography (see

Basic Protocol 1 and Critical Parameters)

NOTE: The ${ }^{1} \mathrm{H}$ NMR and ${ }^{13} \mathrm{C}$ NMR spectra were determined with a $200 \mathrm{MHz}$ Varian Gemini spectrometer with tetramethylsilane as an internal standard. Abbreviations: s, singlet; d, doublet; dd, double doublet; t, triplet; br s, broad signal; m, multiplet; ddd, double doublet of doublet; $\mathrm{dm}$, double multiplet. Liquid secondary-ion (LSIMS) mass spectra were obtained using a KRATOS Concept ${ }^{1} \mathrm{H}$ mass spectrometer.

Synthesis of 


\section{Prepare S.17}

1. Suspend $6.48 \mathrm{~g}(30.0 \mathrm{mmol}) N^{3}$-benzoyluracil, $4.72 \mathrm{~g}$ (20 mmol) S.5, and $7.87 \mathrm{~g}(30.0$ $\mathrm{mmol}$ ) triphenylphosphine in $200 \mathrm{~mL}$ dioxane. Carry out the reaction under a nitrogen atmosphere.

2. Add a solution of $5.90 \mathrm{~mL}(30.0 \mathrm{mmol}) \mathrm{DEAD}$ in $30 \mathrm{~mL}$ dioxane via a dropping funnel over a period of $200 \mathrm{~min}$.

3. Stir the mixture overnight at room temperature.

4. Filter off the precipitate formed during the reaction and wash the precipitate with 50 $\mathrm{mL}$ dioxane.

5. Remove the volatiles of the filtrate by evaporation under vacuum in a rotary evaporator with a vacuum pump.

6. Dissolve the obtained residue in $100 \mathrm{~mL}$ saturated ammonia in methanol.

7. Stir the mixture $90 \mathrm{~min}$ at room temperature.

8. Check the reaction by TLC (see Basic Protocol 1, step 3) using 97.5:2.5 (v/v) dichloromethane/methanol.

9. Remove the volatiles and coevaporate with $20 \mathrm{~mL}$ toluene three times.

10. Purify the residue by column chromatography on a $4 \times 30-\mathrm{cm}$ column using a gradient from $1 \mathrm{~L}$ of $100 \%$ dichloromethane to $1 \mathrm{~L}$ of $97: 3(\mathrm{v} / \mathrm{v})$ dichloromethane/methanol. Combine product-containing fractions and evaporate solvents in vacuo on a rotary evaporator.

The resulting product, $1^{\prime}, 5^{\prime}$-anhydro-4', $6^{\prime}$-O-benzylidene-2', $3^{\prime}$-dideoxy-2'-(uracil-1-yl)-Darabinohexitol (S.17) is obtained in $67 \%$ yield $(4.24 \mathrm{~g}, 12.8 \mathrm{mmol}) . \mathrm{mp}: 200^{\circ} \mathrm{C}$; LSIMS (thygly): $\mathrm{m} / \mathrm{z}: 331[\mathrm{M}+\mathrm{H}]^{+} ;{ }^{l} \mathrm{HNMR}\left(\mathrm{CDCl}_{3}\right): \delta=2.12\left(\mathrm{dt}, 1 \mathrm{H}, 3^{\prime} \mathrm{ax}-\mathrm{H}, \mathrm{J}=11.9 \mathrm{~Hz}, \mathrm{~J}=\right.$ $4.8 \mathrm{~Hz}), 2.52\left(\mathrm{~d}, \mathrm{br}, 1 \mathrm{H}, 3^{\prime}\right.$ eq- $\left.\mathrm{H}, \mathrm{J}=12.9 \mathrm{~Hz}\right), 3.48-3.74\left(\mathrm{~m}, 2 \mathrm{H}, 5^{\prime}-\mathrm{H}, 4^{\prime}-\mathrm{H}\right), 3.79(\mathrm{t}, 1 \mathrm{H}$, $\left.\sigma^{\prime} a x-H, J=10.2 \mathrm{~Hz}\right), 4.06\left(d d, 1 H, l^{\prime} a x-H, J=13.7 \mathrm{~Hz}, J=3.0 \mathrm{~Hz}\right), 4.37\left(d, 1 H, l^{\prime} e q-H\right.$, $J=13.8 \mathrm{~Hz}), 4.36\left(\mathrm{dd}, 1 \mathrm{H}, 6^{\prime} \mathrm{eq}-\mathrm{H}, \mathrm{J}=10 \mathrm{~Hz}, J=4.7 \mathrm{~Hz}\right), 4.78\left(\mathrm{~s}, \mathrm{br}, 1 \mathrm{H}, 2^{\prime}-\mathrm{H}\right), 5.59(\mathrm{~s}$, $1 \mathrm{H}, \mathrm{PhCH}$ ), $5.80(\mathrm{~d}, 1 \mathrm{H}, \mathrm{J}=8.0 \mathrm{~Hz}, 5-\mathrm{H}), 7.34-7.51(\mathrm{~m}, 5 \mathrm{H}, \mathrm{Ph} \mathrm{H}), 8.09(\mathrm{~d}, 1 \mathrm{H}, 6-\mathrm{H}, \mathrm{J}=$ $8.0 \mathrm{~Hz}), 9.30(\mathrm{~s}, \mathrm{br}, 1 \mathrm{H}, \mathrm{NH}) ;{ }^{13} \mathrm{CNMR}\left(\mathrm{CDCl}_{3}\right): \delta=32.8\left(\mathrm{C}-3^{\prime}\right), 51.5\left(\mathrm{C}-2^{\prime}\right), 68.8\left(\mathrm{C}-6^{\prime}\right)$, $68.8\left(C-1^{\prime}\right), 73.6\left(C-4^{\prime}\right), 74.3\left(C-5^{\prime}\right), 102.0,102.2(P h C H, C-5), 126.0(2,6-C P h), 128.4$ (3,5-C Ph), $129.3(4-C P h), 137.1(1-C P h), 142.4(C-6), 150.9(C-2), 163.2(C-4)$.

\section{Prepare S.18}

11. Dissolve $6.23 \mathrm{~g}(96.0 \mathrm{mmol})$ triazole in $120 \mathrm{~mL}$ pyridine.

12. Add $2.61 \mathrm{~mL}(28.0 \mathrm{mmol})$ phosphoroxy chloride and continue stirring at room temperature for $25 \mathrm{~min}$.

13. Add $2.64 \mathrm{~g}(8 \mathrm{mmol})$ S.17.

The solution should turn yellow.

14. Continue stirring $45 \mathrm{~min}$ at room temperature.

15. Remove the volatiles in vacuo on a rotary evaporator and coevaporate with $15 \mathrm{~mL}$ toluene three times.

16. Dissolve the brown residue in $80 \mathrm{~mL}$ dioxane and add $40 \mathrm{~mL}$ of $25 \%$ ammonia.

17. Continue stirring another $30 \mathrm{~min}$.

Synthesis of 1,5-Anhydrohexitol Building Blocks 1.9.18

18. Evaporate to dryness, dissolve in $100 \mathrm{~mL}$ dichloromethane, and wash with $200 \mathrm{~mL}$ water. 
19. Dry the organic layer over sodium sulfate and remove the solvent.

20. Purify the crude product by column chromatography using 95:5 (v/v) dichloromethane/methanol as a solvent. Combine product-containing fractions and evaporate solvent.

The resulting product, $1^{\prime}, 5^{\prime}$-anhydro-4',6'-O-benzylidene-2', $3^{\prime}$-dideoxy-2'-(cytosin-1-yl)$D$-arabinohexitol $(\mathbf{S . 1 8})$ is obtained in $71 \%$ yield $(1.87 \mathrm{~g}, 5.7 \mathrm{mmol}) . \mathrm{mp}: 200^{\circ} \mathrm{C}$; LSIMS (thygly): $\mathrm{m} / \mathrm{z}: 330[\mathrm{M}+\mathrm{H}]^{+},{ }^{l} \mathrm{H} \mathrm{NMR}\left(\mathrm{CDCl}_{3}\right): \delta=2.05\left(\mathrm{dt}, 1 \mathrm{H}, 3^{\prime} \mathrm{ax}-\mathrm{H}, \mathrm{J}=11.9 \mathrm{~Hz}, \mathrm{~J}=\right.$ $4.8 \mathrm{~Hz}), 2.58\left(\mathrm{~d}, \mathrm{br} \mathrm{lH}, 3^{\prime} \mathrm{eq}-\mathrm{H}, \mathrm{J}=12.9 \mathrm{~Hz}\right), 3.43-3.69\left(\mathrm{~m}, 2 \mathrm{H}, 5^{\prime}-\mathrm{H}, 4^{\prime}-\mathrm{H}\right), 3.74(\mathrm{t}, 1 \mathrm{H}$, $\left.\sigma^{\prime} a x-H, J=10.2 \mathrm{~Hz}\right), 4.00\left(\mathrm{dd}, 1 \mathrm{H}, \mathrm{l}^{\prime} \mathrm{ax}-\mathrm{H}, \mathrm{J}=13.7 \mathrm{~Hz}, \mathrm{~J}=3.0 \mathrm{~Hz}\right), 4.20\left(\mathrm{~d}, 1 \mathrm{H}, \mathrm{l}^{\prime} \mathrm{eq}-\mathrm{H}\right.$, $J=13.8 \mathrm{~Hz}), 4.30\left(\mathrm{dd}, 1 \mathrm{H}, \sigma^{\prime} e q-H, J=10 \mathrm{~Hz}, J=4.7 \mathrm{~Hz}\right), 4.82\left(\mathrm{~s}, \mathrm{br}, 1 \mathrm{H}, 2^{\prime}-\mathrm{H}\right), 5.54(\mathrm{~s}$, $1 \mathrm{H}, \mathrm{PhCH}), 5.86(\mathrm{~d}, 1 \mathrm{H}, 5-\mathrm{H}, \mathrm{J}=7.4 \mathrm{~Hz}, 7.30-7.47(\mathrm{~m}, 5 \mathrm{H}$, aromatic $\mathrm{H}), 8.05(\mathrm{~d}, 1 \mathrm{H}, 6-\mathrm{H}$, $J=7.4 \mathrm{~Hz}) ;{ }^{13} \mathrm{C} \mathrm{NMR}\left(\mathrm{CDCl}_{3}\right): \delta=32.5\left(\mathrm{C}-3^{\prime}\right), 51.7\left(\mathrm{C}-2^{\prime}\right), 68.8\left(\mathrm{C}-6^{\prime}\right), 69.2\left(C-1^{\prime}\right), 73.8$ (C-4'), $74.1\left(C-5^{\prime}\right), 94.5(C-5), 101.9(P h C H), 126.0(2,6-C P h), 128.3(3,5-C P h), 129.1$ (4-C Ph), 137.2 (1-C Ph), $143.5(C-6), 156.3(C-2), 162.0(C-4), 165.7(H N C=O)$.

\section{Prepare S.19}

21. Dissolve $2.31 \mathrm{~g}(7.0 \mathrm{mmol}) \mathbf{S . 1 8}$ in $70 \mathrm{~mL}$ pyridine in a $250-\mathrm{mL}$ round-bottom flask equipped with a drying tube.

22. Add $4.1 \mathrm{~mL}$ ( $35.0 \mathrm{mmol}, 5 \mathrm{eq})$ benzoyl chloride and stir the reaction mixture overnight.

23. Analyze by TLC using 95:5 (v/v) dichloromethane/methanol as a solvent.

24. Cool the reaction mixture to $0^{\circ} \mathrm{C}$ and add $10 \mathrm{~mL}$ of $25 \%$ ammonia.

25. Continue stirring $1 \mathrm{hr}$ at room temperature.

26. Remove the volatiles, coevaporate with $15 \mathrm{~mL}$ toluene three times, and dissolve the residue in $100 \mathrm{~mL}$ dichloromethane.

27. Wash with $100 \mathrm{~mL}$ saturated sodium bicarbonate solution, dry the organic layer over sodium sulfate, and remove the solvent in vacuo on a rotary evaporator.

28. Dissolve the resulting brownish foam in $70 \mathrm{~mL}$ dichloromethane.

29. Slowly add $30 \mathrm{~mL}$ TFA and continue stirring $45 \mathrm{~min}$ at room temperature.

30. Monitor by TLC using 9:1 (v/v) dichloromethane/methanol.

31. Evaporate the volatiles and coevaporate with $15 \mathrm{~mL}$ toluene three times.

32. Purify the resulting foam by column chromatography using a $3 \times 35-\mathrm{cm}$ column and a step gradient from 1.5 L of 95:5 to $1.5 \mathrm{~L}$ of 93:7 (v/v) dichloromethane/methanol. Collect product-containing fractions.

33. Reduce volatiles in vacuo on a rotary evaporator to a volume of $10 \mathrm{~mL}$. Precipitate by adding $50 \mathrm{~mL}$ diethyl ether.

The resulting product, $1^{\prime}, 5^{\prime}$-anhydro- $2^{\prime}$ - $\left(N^{4}\right.$-benzoylcytosin-1-yl)-2', $3^{\prime}$-dideoxy-D-arabinohexitol (S.19) is obtained in an overall yield of 67\% $(1.08 \mathrm{~g}, 3.1 \mathrm{mmol}) . \mathrm{mp}: 130^{\circ} \mathrm{C}$; LSIMS (thygly): $\mathrm{m} / \mathrm{z}: 346[\mathrm{M}+\mathrm{H}]^{+} ;{ }^{l} \mathrm{H} \mathrm{NMR}\left(\mathrm{DMSO}-\mathrm{d}_{6}\right): \delta=1.79\left(\mathrm{dt}, 1 \mathrm{H}, 3^{\prime} \mathrm{ax}-\mathrm{H}, \mathrm{J}=13.6 \mathrm{~Hz}\right.$, $J=4.4 \mathrm{~Hz}$ ), $2.20\left(\mathrm{~d}, \mathrm{br}, 1 \mathrm{H}, 3^{\prime}\right.$ eq- $\left.\mathrm{H}, \mathrm{J}=13.7 \mathrm{~Hz}\right), 3.14$ (m, 1H, 5'-H), 3.50-3.70 (m, 3H, $\left.6^{\prime}-H, 4^{\prime}-H\right), 3.81\left(d d, 1 H, l^{\prime} a x-H, J=12.9 H z, J=2.9 H z\right), 4.14\left(d, b r, 1 H, l^{\prime} e q-H, J=\right.$ $13.2 \mathrm{~Hz}), 4.60\left(\mathrm{~m}, 2 \mathrm{H}, 6^{\prime}-\mathrm{OH},+2^{\prime}-H\right), 4.95\left(\mathrm{~d}, 1 \mathrm{H}, 4^{\prime}-\mathrm{H}, \mathrm{J}=5.1 \mathrm{~Hz}\right), 7.31(\mathrm{~d}, 1 \mathrm{H}, 5-\mathrm{H}, \mathrm{J}$ $=7.3 \mathrm{~Hz}), 7.57(\mathrm{~m}, 3 \mathrm{H}$, aromatic $\mathrm{H}), 8.01(\mathrm{~d}, 2 \mathrm{H}$, aromatic $\mathrm{H}, \mathrm{J}=7 \mathrm{~Hz}), 8.50(\mathrm{~d}, 1 \mathrm{H}, 6-\mathrm{H}$, $J=7.4 \mathrm{~Hz}), 11.19(\mathrm{~s}, 1 \mathrm{H}, \mathrm{HNC}=\mathrm{O}) ;{ }^{13} \mathrm{C} \mathrm{NMR}\left(\mathrm{DMSO}-\mathrm{d}_{6}\right): \delta=34.8\left(\mathrm{C}-3^{\prime}\right), 52.3\left(\mathrm{C}-2^{\prime}\right)$, $60.5\left(C-6^{\prime}, C-4^{\prime}\right), 67.2\left(C-1^{\prime}\right), 82.8\left(C-5^{\prime}\right), 95.9(C-5), 128.5(2,3,5,6-C B z), 132.8(1,4-C$ $B z), 148.1$ (C-6), $155.0(C-4), 162.6(C-4), 167.5(H N C=O)$.

Synthesis of Modified Nucleosides

\subsubsection{9}

Supplement 15 
Prepare S.20

34. Dissolve $2.72 \mathrm{~g}(7.9 \mathrm{mmol}) \mathbf{S . 1 9}$ in $40 \mathrm{~mL}$ pyridine and coevaporate three times (40 $\mathrm{mL}$ each).

35. Dissolve in $100 \mathrm{~mL}$ pyridine.

36. Add $4.26 \mathrm{~g}(13.4 \mathrm{mmol})$ of $\mathrm{MMTr} \cdot \mathrm{Cl}$.

37. Stir the reaction at room temperature overnight.

38. Monitor by TLC using 95:5 (v/v) dichloromethane/methanol.

39. Quench the reaction with $200 \mathrm{~mL}$ saturated sodium bicarbonate solution.

40. Extract twice with $200 \mathrm{~mL}$ dichloromethane.

41. Dry the organic layer over sodium sulfate, evaporate the solvent, and coevaporate three times with $20 \mathrm{~mL}$ toluene.

42. Purify the residue by column chromatography on a $3 \times 35-\mathrm{cm}$ column using a step gradient of $1 \mathrm{~L}$ each 99:1, 98:2, 97:3, and 96:4 (v/v) dichloromethane/methanol. Combine product-containing fractions and evaporate solvents.

The resulting product, $1^{\prime}, 5^{\prime}$-anhydro-2'-( $N^{4}$-benzoylcytosin-1-yl)-2', $3^{\prime}$-dideoxy- $6^{\prime}$ monomethoxytrityl-D-arabinohexitol (S.20) is obtained in $80 \%$ yield $(3.88 \mathrm{~g}, 6.3 \mathrm{mmol}$ ). mp: $140^{\circ} \mathrm{C} ; \mathrm{LSIMS}$ (thygly/ NaOAc): $\mathrm{m} / z: 640[\mathrm{M}+\mathrm{Na}]^{+} ;{ }^{1} \mathrm{H} \mathrm{NMR}\left(\mathrm{CDCl}_{3}\right): \delta=1.90(\mathrm{~m}$, $\left.1 \mathrm{H}, 3^{\prime} \mathrm{ax}-\mathrm{H}\right), 2.55\left(\mathrm{br}, \mathrm{d}, 1 \mathrm{H}, 3^{\prime} \mathrm{eq}-\mathrm{H}, \mathrm{J}=12.2 \mathrm{~Hz}\right), 3.08\left(\mathrm{~s}, \mathrm{br}, 1 \mathrm{H}, 5^{\prime}-\mathrm{H}\right), 3.42\left(\mathrm{~m}, 3 \mathrm{H}, 4^{\prime} \mathrm{H}\right.$, $\left.6^{\prime}-H\right), 3.79\left(s, 3 H, O_{3}\right), 3.89\left(d d, 1 H, l^{\prime} a x-H, J=13.5 \mathrm{~Hz}, J=2.9 \mathrm{~Hz}\right), 4.01(\mathrm{~s}, \mathrm{br}, 1 \mathrm{H}$, $\left.4^{\prime}-\mathrm{OH}\right), 4.25\left(\mathrm{~d}, \mathrm{br}, 1 \mathrm{H}, \mathrm{l}^{\prime}\right.$ eq- $\left.\mathrm{H}, \mathrm{J}=13.5 \mathrm{~Hz}\right), 4.83\left(\mathrm{~s}, \mathrm{br}, 1 \mathrm{H}, \mathrm{2}^{\prime}-\mathrm{H}\right), 6.85(\mathrm{~d}, 2 \mathrm{H}$, aromatic $H, J=8.8 \mathrm{~Hz}), 7.20-7.70(\mathrm{~m}, 18 \mathrm{H}$, aromatic $\mathrm{H}+5-\mathrm{H}), 8.73(\mathrm{br}, \mathrm{d}, 2 \mathrm{H}, \mathrm{NH}+6-\mathrm{H}, \mathrm{J}=7.6$ $\mathrm{Hz}) ;{ }^{13} \mathrm{CNMR}\left(\mathrm{CDCl}_{3}\right): \delta=35.0\left(\mathrm{C}-3^{\prime}\right), 52.7\left(\mathrm{C}-2^{\prime}\right), 55.1\left(\mathrm{OCH}_{3}\right), 62.1\left(\mathrm{C}-4^{\prime}\right), 62.7\left(\mathrm{C}-6^{\prime}\right)$, $68.4\left(C-1^{\prime}\right), 80.9\left(C-5^{\prime}\right), 86.8(O C T r), 96.4(C-5), 113.2\left(3^{\prime} 5^{\prime} C\right), 127.1(4 C, 2 \times), 127.9$ (3,5C Bz), $128.0(2,6 C 2 \times), 128.4(3,5 C 2 \times), 128.9(2,6 C \mathrm{Bz}), 130.0\left(2^{\prime} 6^{\prime} \mathrm{C}\right), 132.7(4 \mathrm{C}$ $B z), 132.9(1 C B z), 135.0\left(1^{\prime} \mathrm{C}\right), 143.9(1 C 2 \times), 148.0(C-6), 155.5(C-2), 158.6\left(4^{\prime} \mathrm{C}\right), 161.9$ $(C-4), 166.3(H N C=O)$.

\section{COMMENTARY}

\section{Background Information}

Hexitol nucleic acid (HNA) is the first example of an oligonucleotide with a six-membered carbohydrate moiety that hybridizes with natural nucleic acids. The base moiety is connected to the 2'-position of the sugar, which means that the nucleic acids have no anomeric center. These nucleic acids are stable against enzymatic and chemical degradation (Hendrix et al., 1997a,b). HNA can be synthesized by the phosphoramidite method (Hendrix et al., 1997a,b; also see UNIT 3.3) starting from the building blocks described in this protocol.

HNAs are RNA-selective oligonucleotides, which means that they hybridize more strongly to RNA as the complement than to DNA (Hendrix et al., 1997a,b). The HNA-RNA duplex is thermally more stable than the DNA-RNA duplex, making HNA suitable as a steric blocking agent for antisense purposes $\left(\Delta T_{\mathrm{m}} / \mathrm{mod}+1^{\circ}\right.$ to $+5^{\circ} \mathrm{C}$ ). This higher stability is mainly based on its preorganization (entropy factor).
The NMR structure of an HNA-RNA duplex has been solved (Lescrinier et al., 2000a,b) and it shows typical A-form geometry. The hexitol nucleoside itself is a good mimic of a furanose nucleoside in its $2^{\prime}$-exo, $3^{\prime}$-endo conformation (northern type). In a qualitative model, cellular uptake of HNA seems to be similar to that of phoshorothioate oligonucleotides, using cationic lipids as a transfecting agent (Atkins et al., 2000).

Hybridization of HNA with RNA is more sequence specific than between DNA and RNA, which is demonstrated by the larger difference in $T_{\mathrm{m}}$ between match and mismatch duplexes of HNA-RNA versus DNA-RNA (Hendrix et al., 1997b).

HNA functions as a very efficient template for nonenzymatic oligomerization of activated ribonucleoside monophosphates (Kozlov et al., 1999a,b). HNA templates are chiral-discriminating in that they selectively accept D-nucleotides over L-nucleotides for primer extension 
(Kozlov et al., 1999a,b). Two HNA codons (6 nucleotides) incorporated in an otherwise intact RNA are accepted as messenger in the translation process (Lavrik et al., 2001). Hexitol nucleoside triphosphates may be used by polymerases for enzymatic incorporation into oligodeoxyribonucleotides (Vastmans et al., 2000).

\section{Critical Parameters}

Overall, it is important that for each step of the syntheses the starting sugar- and basebuilding blocks or nucleosides, respectively, are thoroughly dried either by coevaporation with anhydrous pyridine or in a desiccator over phosphorus pentoxide. The MMtr-protected building blocks (S.9, S.13, S.16, and S.20) are dried over silica blue (not over phosphorous pentoxide) and can be stored at $4^{\circ} \mathrm{C}$.

For all reactions using anhydrous solvents, the glassware must be predried at $70^{\circ} \mathrm{C}$ for at least $2 \mathrm{hr}$.

All solvents must be distilled before use. Anhydrous solvents are very important. They should either be freshly distilled and stored under nitrogen or argon, or be taken from a freshly opened bottle of commercially prepared anhydrous solvent.

For evaporation of solvents, it is helpful if the rotary evaporator is equipped with a dry ice condenser.

For all compounds, a sample of $100 \mathrm{mg}$ should be kept as a reference.

In all cases, the reaction progress is followed by TLC. The starting material ( $1 \mathrm{mg}$ diluted to $200 \mu \mathrm{L})$ as well as the reaction mixture (100 $\mu \mathrm{L})$ are prepared. A baseline is marked and the spots of the solutions are placed at equal distances, with the reaction mixture in the middle surrounded by starting material. After developing in the appropriate solvent, the end line of the TLC is marked, and spots are identified first under a UV lamp (254 $\mathrm{nm}$ ) and then by spraying the plates with anisaldehyde/sulfuric acid spray and heating to $150^{\circ} \mathrm{C}$. The sugar-containing products turn blue. The monomethoxytritylated products (S.9, S.13, S.16, and S.20) turn yellow immediately after spraying (deprotection). After heating, the product-containing spots turn green.

Before each column purification, TLC is performed on the reaction mixture to evaluate the completeness of the reaction and the presence of starting materials to be recovered. Silica gel $(0.060$ to $0.200 \mathrm{~nm})$ is suspended in the first solvent and loaded into the column, which is tightly packed by gentle tapping. The silica layer is topped by a $1-\mathrm{cm}$ layer of sand. The crude product is dissolved in a minimum volume of solvent and applied to the column. The fractions are collected with a fraction collector and a roster of the collector is drawn on a TLC plate. A drop from each fraction is applied to the corresponding field on the plate, and the plates are evaluated under a UV lamp and by applying anisaldehyde/sulfuric acid spray. The fraction-containing products (or unreacted starting material) are tested for homogeneity by TLC. Elution of the column is continued with the final solvent of the indicated gradient until all product has been isolated. Product-containing fractions are combined and solvents are removed in vacuo on a rotary evaporator.

\section{Troubleshooting}

\section{Preparation of $h C$}

The identical product can be obtained by Mitsunobu reaction of $N^{4}$-benzoylcytosine with the hexitol building block S.5 as demonstrated in De Bouvere et al. (1997). In this case the yield was rather modest $(34 \%)$ and turned out to be less reproducible. Furthermore, in some attempts the $1^{\prime}, 5^{\prime}$-anhydro- $2^{\prime}$ - $\left(N^{4}\right.$-benzoylcytosin-2-yl)-4', $6^{\prime}$ - $O$-benzylidene- $2^{\prime}, 3^{\prime}$ dideoxy-D-arabinohexitol ( $O$-nucleoside) instead of the desired $1^{\prime}, 5^{\prime}$-anhydro- $2^{\prime}-\left(N^{4}\right.$ benzoylcytosin-1-yl)-4',6'-O-benzylidene-2', $3^{\prime}$-dideoxy-D-arabinohexitol ( $N$-nucleoside) was obtained.

\section{Preparation of $h G$}

Due to the high price of 6-iodo- $9 H$-purine2 -amine, the synthesis of $h \mathrm{G}$ may be performed using the Mitsunobu reaction of 6-chloro- $9 \mathrm{H}$ purine-2-amine with the hexitol building block S.5. If 6-iodo-9H-purine-2-amine is available, another approach for obtaining $h \mathrm{G}$ is described in De Bouvere et al. (1997).

\section{Anticipated Results}

Preparation of appropriately protected anhydrohexitol building blocks is straightforward using the described protocols. Automated oligomer assembly using standard phosphoramidite chemistry cycles - as provided by the manufacturers of automated DNA synthesizers—will yield anhydrohexitol oligomers.

\section{Time Considerations}

In the planning of the syntheses it has to be considered that most of reactions have to be stirred overnight. Normally each step (includ-
Synthesis of Modified Nucleosides

\subsubsection{1}

Supplement 14 
ing purification and spectroscopic analysis) can be performed in 1.5 to 2 working days.

\section{Literature Cited}

Atkins, D., Miller, M., De Bouvere, B., Van Aerschot, A., and Herdewijn, P. 2000. Evaluation of the cellular uptake of hexitol nucleic acids in HeLa cells. Pharmazie 55:615-617.

De Bouvere, P., Kerremans, L., Rozenski, J., Janssen, G., Van Aerschot A., Claes, P., Busson, R., and Herdewijn, P. 1997. Improved synthesis of anhydrohexitol building blocks for oligonucleotide synthesis. Liebigs Ann./Recueil 14531461.

De Winter, H., Lescrinier, E., Van Aerschot, A., and Herdewijn, P. 1998. Molecular dynamics simulation to investigate differences in minor groove hydration of HNA/RNA hybrids as compared to HNA/DNA complexes. J. Am. Chem. Soc. 120:5381-5394.

Hendrix, C., Verheggen, I., Rosemeyer, H., Seela, F., Van Aerschot, A., and Herdewijn, P. 1997a. 1',5'Anhydrohexitol oligonucleotides: Synthesis, base pairing and recognition by regular oligodeoxyribonucleotides and oligoribonucleotides. Chem. Eur. J. 3:110-119.

Hendrix, C., Rosemeyer, H., De Bouvere, B., Van Aerschot A., Seela, F., and Herdewijn, P. 1997b. $1^{\prime}, 5^{\prime}$-Anhydrohexitol oligonucleotides: Hybridization and strand displacement with oligoribonucleotides, interaction with RNase $\mathrm{H}$ and HIV reverse transcriptase. Chem. Eur.J. 3:15131520.

Kocienski, P. and Pant, C. 1982. A convenient preparation of some 2,3,4,6-tetraacetyl-1,5-anhydroD-hexitols. Carbohydr. Res. 110:330-332.

Kozlov, I.A., Politis, P.K., Pitsch, S., Herdewijn, P., and Orgel, L.E. 1999a. A highly enantio-selective hexitol nucleic acid template for nonenzymatic oligoguanylate synthesis. J. Am. Chem. Soc. 121:1108-1109.
Kozlov, I.A., Politis, P.K., Van Aerschot, A., Busson, R., Herdewijn, P., and Orgel, L.E. 1999b. Nonenzymatic synthesis of RNA and DNA oligomers on hexitol nucleic acid templates: The importance of the A structure. J. Am. Chem. Soc. 121:2613-2656.

Lavrik, I.N., Avdeeva, O.N., Dontsova, O.A., Froeyen, M., and Herdewijn, P.A. 2001. Translational properties of mHNA, a messenger RNA containing anhydrohexitol nucleotides. Biochemistry 40:11777-11784.

Lescrinier, E., Esnouf, R.M., Schraml, J., Busson, R., and Herdewijn, P. 2000a. Solution structure of a hexitol nucleic acid duplex with four consecutive TT base pairs. Helv. Chim. Acta 83:1291-1310.

Lescrinier, E., Esnouf, R.M., Schraml, J., Busson, R., Heus, H.A., Hilbers, C.W., and Herdewijn, P. 2000b. Solution structure of a HNA-RNA hybrid. Chem. \& Biol. 7:719-731.

Vastmans, K., Pochet, S., Peys, A., Kerremans, L., Van Aerschot, A., Hendrix, C., Marlière, P., and Herdewijn, P. 2000. Enzymatic incorporation in DNA of 1,5-anhydrohexitol nucleotides. Biochemistry 39:12757-12765.

Verheggen, I., Van Aerschot, A., Toppet, S. Snoeck, R., Janssen, G., Balzarini, J., De Clercq, E., and Herdewijn, P. 1993. Synthesis and antiherpes virus activity of 1,5-anhydrohexitol nucleosides. J. Med. Chem. 36:2033-2039.

Verheggen, I., Van Aerschot, A., Van Meervelt, L., Rozenski, J., Wiebe, L., Snoek, R., Andrei, G., Balzarini, J., Claes, P., De Clercq, E., and Herdewijn, P. 1995. Synthesis, biological evaluation, and structure analysis of a series of new 1,5-anhydrohexitol nucleosides J. Med. Chem. 38:826835 .

Contributed by Irene M. Lagoja, Arnaud Marchand, Arthur Van Aerschot, and Piet Herdewijn

Rega Institute for Medical Research Leuven, Belgium
Synthesis of 1,5-Anhydrohexitol Building Blocks 